CONFORMAL GEOMETRY AND DYNAMICS

An Electronic Journal of the American Mathematical Society

Volume 9, Pages 46-75 (April 26, 2005)

S $1088-4173(05) 00106-2$

\title{
TRACE COORDINATES OF TEICHMÜLLER SPACE OF RIEMANN SURFACES OF SIGNATURE $(0,4)$
}

\author{
THOMAS GAUGLHOFER AND KLAUS-DIETER SEMMLER
}

\begin{abstract}
We explicitly give $\mathcal{T}$, the Teichmüller space of four-holed spheres (which we call X pieces) in trace coordinates, as well as its modular group and a fundamental domain for the action of this group on $\mathcal{T}$ which is its moduli space. As a consequence, we see that on any hyperbolic Riemann surface, two closed geodesics of lengths smaller than $2 \operatorname{arccosh}(2)$ intersect at most once; two closed geodesics of lengths smaller than $2 \operatorname{arccosh}(3)$ are both non-dividing or intersect at most once.
\end{abstract}

\section{INTRODUCTION}

This paper deals with Teichmüller space and the Riemann moduli problem for surfaces of signature $(0,4)$ which we call X pieces. The Riemann moduli problem is to describe the space of isomorphism classes of Riemann surfaces of a given signature which is known as the moduli space. Teichmüller space can be defined in many different ways, for instance as the space of isotopy classes of marked complex structures of the Riemann surface, where two structures define the same point in Teichmüller space if there exists a holomorphic homeomorphism homotopic to the identity leading from one to the other. Once Teichmüller space is well known, we can construct the modular group or mapping class group and build the quotient of Teichmüller space with it to get the moduli space.

In this paper we take the view that a Riemann surface is given by a Fuchsian group, namely a discrete subgroup of $S L(2, \mathbb{R})$ acting on the upper half plane $\mathbb{H}$ by Möbius transformations and by which we quotient $\mathbb{H}$ to obtain the structure, which is determined by this subgroup up to conjugation. We choose $S L(2, \mathbb{R})$ rather than $P S L(2, \mathbb{R})$, as is usually done in literature, because this gives us additional information on direction of geodesics (see section 2). However, as we choose the traces of the generating elements to be positive, these two approaches are equivalent (see [SS92]). In this language we can define Teichmüller space as the space of endomorphisms of the Fuchsian subgroup into $S L(2, \mathbb{R})$ up to conjugation that preserve parabolic elements and whose image is discrete.

As the referee pointed out, this approach is quite the same as the one taken in Kee77. (based upon previous work; see Kee65, Kee66, Kee71, Kee73]) where Keen gives rough fundamental domains for the action of modular groups on Teichmüller spaces for various signatures including $(0,4)$. However, our way to show that there

Received by the editors September 3, 2003 and, in revised form, February 8, 2005.

2000 Mathematics Subject Classification. Primary 32G15, 30F35; Secondary 11F06.

The authors were supported in part by the Swiss National Science Foundation, SNSF Grant \#2100-065270, Teichmüller Spaces in Trace coordinates and Modular groups.

(C)2005 American Mathematical Society Reverts to public domain 28 years from publication 
are no two points in our fundamental domain that correspond to isometric surfaces (based upon shortest dividing geodesics; see sections 5 and 6 ) is quite different from the techniques developed by Keen.

Another approach would have been to consider the characters of representation of the fundamental group of a four-holed sphere into $S L(2, \mathbb{R})$ as this is done in [BG99], based upon [Mag80, where Magnus treats rings of Fricke characters of representations of free groups on $n$ free generators into $S L(2, \mathbb{C})$ and their automorphisms. However, this approach has two disadvantages: Firstly, the modular group (group of automorphisms) of the ring of Fricke characters is not the modular group acting on Teichmüller space of surfaces of signature $(0, n+1)$ because $n$ free generators do not necessarily generate such a surface. Indeed, the fundamental group of surfaces of signature $(0, n+1)$ is a free group on $n$ generators, isomorphic to the one of surfaces of signature $(1, n-1)$, yet these surfaces aren't homeomorphic. Secondly, the approach wouldn't explicitly give the subgroup of $S L(2, \mathbb{R})$ isomorphic to the fundamental group of the surface nor its action on the upper half plane and we couldn't explicitly give fundamental domains for four-holed spheres.

Yet a different point of view is taken in [Lu098] where Feng Luo gives a set of conditions for a function $f$ over the isotopy classes of essential unoriented simple closed curves on an orientable surface to be the geodesic length function of a hyperbolic metric on the surface. As these conditions are polynomial equations in $\cosh (f / 2)$, the parameterization of Teichmüller space is in the case of an X piece the same as the one we obtained (up to a factor of 2 because we choose half-traces), but uses heavy machinery such as the Maskit combination theorem (cf. [Mas65, Mas88]) and doesn't give the explicit parameterization of the fundamental group of X pieces as a subgroup of $S L(2, \mathbb{R})$ which is essential in order to give the modular group and solve the Riemann moduli problem in the second part of this paper.

The main results of this paper are the explicit parameterization of the fundamental group of X pieces as a subgroup of $S L(2, \mathbb{R})$ and of Teichmüller space $\mathcal{T}$ as a subset of a hypersurface in $\mathbb{R}^{7}$ in terms of traces (actually half of the traces of the matrices), the fact that the modular group in these trace coordinates is polynomial, and the explicit description of moduli space:

Theorem A. The subgroup of $S L(2, \mathbb{R})$ acting on the upper half plane $\mathbb{H}$ by Möbius transformations such that the quotient surface has signature $(0,4)$, is freely generated by $\alpha, \beta$ and $\delta$ or a simultaneous conjugation of these three:

$$
\begin{aligned}
& \alpha:=\quad\left(\begin{array}{cc}
a-\frac{a c+b}{\sqrt{c^{2}-1}} & -\frac{1}{c^{2}-1} \\
h(a, b, c) & a+\frac{a c+b}{\sqrt{c^{2}-1}}
\end{array}\right), \\
& \beta:=\left(\begin{array}{cc}
b-\frac{b c+a}{\sqrt{c^{2}-1}} & -\frac{c+\sqrt{c^{2}-1}}{c^{2}-1} \\
h(a, b, c)\left(c-\sqrt{c^{2}-1}\right) & b+\frac{b c+a}{\sqrt{c^{2}-1}}
\end{array}\right), \\
& \delta:=\left(\begin{array}{cc}
d+\frac{c d+e}{\sqrt{c^{2}-1}} & -\frac{p\left(c+\sqrt{c^{2}-1}\right)}{c^{2}-1} \\
\frac{h(c, d, e)\left(c-\sqrt{c^{2}-1}\right)}{p} & d-\frac{c d+e}{\sqrt{c^{2}-1}}
\end{array}\right),
\end{aligned}
$$


with

$$
\begin{aligned}
& p:=-\frac{a e+b d+(a d+b e)\left(c-\sqrt{c^{2}-1}\right)+\sqrt{c^{2}-1}\left(\left(c-\sqrt{c^{2}-1}\right) z-x\right)}{h(a, b, c)}, \\
& h(u, v, w):=u^{2}+v^{2}+w^{2}+2 u v w-1 .
\end{aligned}
$$

Where $(a, b, c, d, e, x, z)$ is an element of Teichmüller space $\mathcal{T}$ of surfaces of signature $(0,4)$ in trace coordinates, i.e.

$$
\mathcal{T}:=\{(a, b, c, d, e, x, z) \in \mathcal{P} \mid Q=0\}
$$

with the parameter space

$$
\mathcal{P}:=\left\{(a, b, c, d, e, x, z) \in \mathbb{R}^{7} \mid a, b, c, d, e, x, z>1\right\}
$$

and the polynomial

$$
\begin{aligned}
Q:= & a^{2}+b^{2}+c^{2}+d^{2}+e^{2}+x^{2}+z^{2}+4 a b d e-1 \\
& +2 c(a b+d e)+2 x(a d+b e)+2 z(a e+b d)-2 c x z=0 .
\end{aligned}
$$

Remark. The polynomial $Q$ and its null-set have already been studied in [BG99; however, the authors didn't give Teichmüller space for four-holed spheres in that paper. Nevertheless, the result regarding the parameterization of $\mathcal{T}$ is already given in Luo98, where Feng Luo discusses more general results (previous related results can be found also in [SS89, SS88 SS86]) and thus not new. However, our method of computation is less complicated and allows us to give the explicit matrices generating the fundamental group.

Theorem B. The modular group of Teichmüller space $\mathcal{T}$ of surfaces of signature $(0,4)$ acts polynomially on $\mathcal{T}$ in these trace coordinates.

Theorem C. The set of isometry classes of surfaces of signature $(0,4)$ is in a 1-1-correspondence with the set

$$
\begin{aligned}
\mathcal{F}:=\left\{(a, b, c, d, e, x, z) \in \mathbb{R}^{7} \mid \begin{array}{l}
1<a \leq \min \{b, d, e\}, \\
1<c \leq z \leq x \leq c z-a d-b e
\end{array}\right. \\
\text { and } Q=0\} .
\end{aligned}
$$

The algebraic formalism using quaternions introduced in section 2 leads to the building blocks (pairs of pants or Y pieces; section 3) of the X piece and allows us to determine explicitly a fundamental domain for the $\mathrm{X}$ piece and its fundamental group as a subgroup of $S L(2, \mathbb{R})$ (section 4$)$. We then parameterize Teichmüller space $\mathcal{T}$ of these four-holed spheres in terms of traces. In section 5 , we show that the modular group in trace coordinates is polynomial and in section 6 we treat the moduli problem by explicitly giving the moduli space, seen as a fundamental domain for the modular group of $\mathcal{T}$. Finally, in the last two sections, we give some motivation for the approach that uses trace coordinates: We show that in classical Fenchel-Nielsen coordinates, the modular group gets extremely complicated, and give a general result about geodesic lengths on Riemann surfaces.

\section{Preliminaries}

In this section we recall an algebraic formalism studied in [Sem88] to treat geometric objects as well as isometries of the upper half plane $\mathbb{H}$. 
We consider Möbius transformations leaving the upper half plane invariant, and parameterize them by elements of $S L(2, \mathbb{R}) \subset M(2, \mathbb{R})$, a vector space over $\mathbb{R}$ which we give the basis of quaternions $\{\mathbf{1}, \mathbf{I}, \mathbf{J}, \mathbf{K}\}$ :

$$
\mathbf{1}=\left(\begin{array}{ll}
1 & 0 \\
0 & 1
\end{array}\right), \mathbf{I}=\left(\begin{array}{rr}
1 & 0 \\
0 & -1
\end{array}\right), \mathbf{J}=\left(\begin{array}{ll}
0 & 1 \\
1 & 0
\end{array}\right), \mathbf{K}=\left(\begin{array}{rr}
0 & 1 \\
-1 & 0
\end{array}\right) .
$$

Notation. We call $H_{0}$ the vector subspace generated by $\mathbf{I}, \mathbf{J}$ and $\mathbf{K}$. We call trace of an element $\alpha$ of $S L(2, \mathbb{R})$ its 1-component and note it $\operatorname{tr}(\alpha)$ (it is actually half of the standard trace of the matrix $\alpha)$. If an element of $S L(2, \mathbb{R})$ is written as $(a+A)$ we mean the element $a \mathbf{1}+A$, with $a \in \mathbb{R}$ and $A \in H_{0}$.

\subsection{The products.}

Definition 1. The "scalar" product over $H_{0}$ is the symmetric bilinear form

$$
(., .): H_{0} \times H_{0} \rightarrow \mathbb{R} \text { defined by }(A, B)=a_{1} b_{1}+a_{2} b_{2}-a_{3} b_{3}
$$

where $A$ and $B$ are the matrices without traces: $A=a_{1} \mathbf{I}+a_{2} \mathbf{J}+a_{3} \mathbf{K}$ and $B=b_{1} \mathbf{I}+b_{2} \mathbf{J}+b_{3} \mathbf{K}$.

Definition 2. The $\wedge$-product is the unique antisymmetric bilinear form $\wedge: H_{0} \times H_{0} \rightarrow H_{0}$ satisfying $\mathbf{I} \wedge \mathbf{J}=\mathbf{K}, \mathbf{I} \wedge \mathbf{K}=\mathbf{J}$ and $\mathbf{J} \wedge \mathbf{K}=-\mathbf{I}$, i.e. $A \wedge B=\left(a_{3} b_{2}-a_{2} b_{3}\right) \mathbf{I}+\left(a_{1} b_{3}-a_{3} b_{1}\right) \mathbf{J}+\left(a_{1} b_{2}-a_{2} b_{1}\right) \mathbf{K}$.

Remark. The usual matrix product of two elements $(a+A)$ and $(b+B)$ of $S L(2, \mathbb{R})$ can be written as

$$
(a+A) *(b+B)=a b+(A, B)+a B+b A+A \wedge B .
$$

2.2. The geometric elements of $S L(2, \mathbb{R})$. At first sight, the products in 2.1 operate on matrices only and have no geometric meaning. Using Möbius transformations we can give them a geometric sense:

Definition 3. Let $\alpha=(a+A) \in S L(2, \mathbb{R}), A=a_{1} \mathbf{I}+a_{2} \mathbf{J}+a_{3} \mathbf{K}$ and $m_{\alpha}$ the Möbius transformation $z \mapsto \frac{\left(a+a_{1}\right) z+\left(a_{2}+a_{3}\right)}{\left(a_{2}-a_{3}\right) z+\left(a-a_{1}\right)}$. We define the geometric object corresponding to $(a+A)$ :

- if $(A, A)>0$, the geodesic passing through the fixed points of $m_{\alpha}$;

- if $(A, A)=0$, the (infinite) fixed point of $m_{\alpha}$;

- if $(A, A)<0$, the fixed point of $m_{\alpha}$ that has a positive imaginary part.

Remark. A half-circle centred on the real axis is indeed a geodesic of the upper half plane endowed with the distance $d$, defined by $\cosh (d(A, B))=\frac{|(A, B)|}{\sqrt{(A, A)(B, B)}}$ for $(a+A),(b+B) \in S L(2, \mathbb{R})$ such that $(A, A)<0$ and $(B, B)<0$ (Sem88).

Definition 4. If $(a+A) \in S L(2, \mathbb{R})$ is of positive trace and $(A, A)>0$, we give its corresponding geodesic the following direction:

- if $a_{2}-a_{3}>0$, the geodesic is directed towards the right,

- if $a_{2}-a_{3}<0$, towards the left,

- if $a_{2}-a_{3}=0$ and $\left|a+a_{1}\right|>1$, upwards,

- if $a_{2}-a_{3}=0$ and $\left|a+a_{1}\right|<1$, downwards.

If $(a+A) \in S L(2, \mathbb{R})$ is of negative trace and $(A, A)>0$, we give its corresponding geodesic the direction of the geodesic $(-a-A)$ defined as before. 
Remark. Note that the orientation of a geodesic corresponding to $(a+A) \in S L(2, \mathbb{R})$, $a<0$ is well defined because $(a+A)$ and $(-a-A)$ correspond to the same Möbius transformation.

This definition gives a geodesic that is directed from the repulsive towards the attractive fixed point of $m_{\alpha}$ if $\operatorname{tr}(\alpha)>0$.

Note also that $(a+A)$ and $(a+A)^{-1}=(a-A)$ correspond to the same geodesic but have opposite orientation.

2.3. Isometries of $\mathbb{H}$. Up to now we treated the correspondence between matrices $(\in S L(2, \mathbb{R}))$ and geometric objects of the upper half plane $\mathbb{H}$. But we know that there is a homomorphism of groups from $S L(2, \mathbb{R})$ to Möbius transformations. We can therefore consider the action of a geodesic, represented by a matrix, on the upper half plane.

Definition 5. For each matrix $\alpha=(a+A) \in S L(2, \mathbb{R})$ we define the homomorphism,

$$
h_{\alpha}: S L(2, \mathbb{R}) \rightarrow S L(2, \mathbb{R}): \beta \mapsto \alpha * \beta * \alpha^{-1} .
$$

Proposition 6. The restriction of $h_{\alpha}$ to $\{(b+B) \in S L(2, \mathbb{R}) \mid 0<b<1\}$ is also an homomorphism $\left(0<\operatorname{tr}\left(h_{\alpha}(\beta)\right)<1\right)$ and even an isometry of $\mathbb{H}$.

Proof. We have $\operatorname{tr}\left(h_{\alpha}(b+B)\right)=b$ by direct calculation using $(a+A)^{-1}=(a-A)$. Thus $h_{\alpha}(b+B)=b+\alpha * B * \alpha^{-1}$. As $(B, C)=\operatorname{tr}(B * C)$ we have also

$$
\begin{aligned}
(B, C) & =\operatorname{tr}(B * C) & & \operatorname{tr}\left(h_{\alpha}(B * C)\right) \\
& =\operatorname{tr}\left(\alpha * B * C * \alpha^{-1}\right) & & \operatorname{tr}\left(\alpha * B * \alpha^{-1} * \alpha * C * \alpha^{-1}\right) \\
& =\left(\alpha * B * \alpha^{-1}, \alpha * C * \alpha^{-1}\right) & = & \left(h_{\alpha}(B), h_{\alpha}(C)\right) .
\end{aligned}
$$

Therefore $h_{\alpha}$ leaves the "scalar" product invariant and thus is an isometry.

Remark. This isometry $h_{\alpha}$, acting on matrices, is actually the same transformation as the Möbius transformation $m_{\alpha}$ acting on the points of the upper half plane; in the sense that if $\gamma=(c+C)$ corresponds to the point $z$ in $\mathbb{H}$, then the matrix $h_{\alpha}(\gamma)$ corresponds to the point $m_{\alpha}(z)$ and $h_{\alpha} \circ h_{\beta}(\gamma)=h_{\alpha * \beta}(\gamma)$ corresponds to $m_{\alpha}\left(m_{\beta}(x)\right)$.

\section{THE Y PIECE}

A lot of research has been done on the Riemann surface of signature $(0,3)$ also called pair of pants, three-holed sphere or Y piece (e.g. Bea83, Bin00, Bus92, Mas88, SS92]). Here we will only give two results that concern geodesics on a Y piece.

Lemma 7. Let $\alpha=(a+A)$ and $\beta=(b+B) \in S L(2, \mathbb{R})$ with $a>1$ and $b>1$ satisfying $H_{Y}$, i.e. be such that there is $\gamma=(c+C) \in S L(2, \mathbb{R})$ with $c>1$ and $\alpha * \beta * \gamma=-1$.

Then the geodesics corresponding to $\alpha, \beta$ and $\gamma=-(\alpha * \beta)^{-1}$ form a domain whose borders are three geodesics that don't intersect, i.e. they are disjoint and no one of them separates $\mathbb{H}$ into two regions that contain each one of the others.

Proof. See for instance [Bin00]. 
Proposition 8. If $\alpha$ and $\beta$ satisfy $H_{Y}$, then there exist only two situations for the hyperbolic triangle formed by $\alpha, \beta$ and $\gamma=-(\alpha * \beta)^{-1}$ in the unit disc model 1 of $\mathbb{H}$ :

(A) The triangle is positively oriented and the names of the axes are as follows:

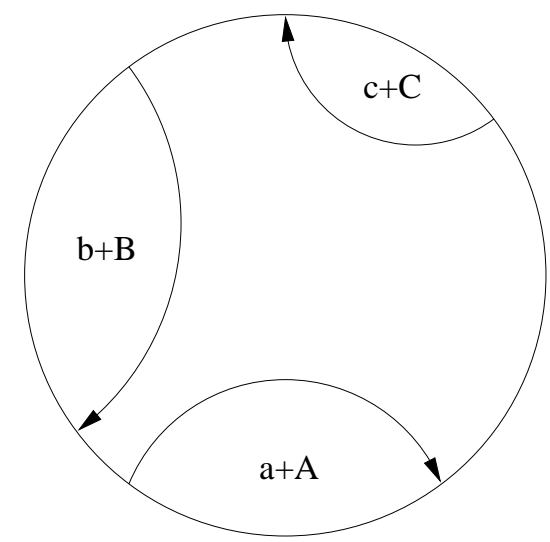

(B) The triangle is negatively oriented and the names of the axes are as follows:

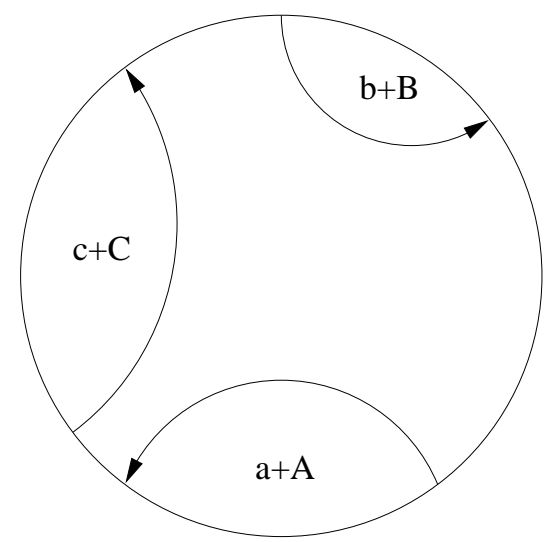

Proof. By the previous lemma we know that the axes form a triangle.

Let two of the "edges" (axes) be positively oriented. We can suppose that their names are $(a+A)$ and $(b+B)$ by cyclic permutation of the names. We can also suppose that (in the upper half-plane model) they are both directed towards the right and that $(a+A)$ is on the left of $(b+B)\left(\frac{a_{1}}{a_{2}-a_{3}}<\frac{b_{1}}{b_{2}-b_{3}}\right)$ by conjugation 2. But in this case we have $\frac{c_{2}-c_{3}}{\left(a_{2}-a_{3}\right)\left(b_{2}-b_{3}\right)}=\frac{a}{a_{2}-a_{3}}-\frac{a_{1}}{a_{2}-a_{3}}+\frac{b}{b_{2}-b_{3}}+\frac{b_{1}}{b_{2}-b_{3}}>\frac{a}{a_{2}-a_{3}}+\frac{b}{b_{2}-b_{3}}$ $>0$ and $(c+C)$ is also directed towards the right. But this means that we are in situation (A) because in all other cases we can construct (by cyclic permutation of the names and conjugation) a case where $(c+C)$ is directed towards the left.

Analogously, we are in situation (B) if two of the "edges" are negatively oriented.

\footnotetext{
${ }^{1}$ If we choose an arbitrary point $p$ of the unit circle, then there exists a natural isometry between $\mathbb{D}^{2} \backslash\{p\}$ and $\mathbb{H}$ such that $\mathbb{S} \backslash\{p\}$ is mapped to $\mathbb{R}$.

${ }^{2}$ choice of $p$
} 
Proposition 9. The domain whose boundaries $s_{1}, \ldots, s_{4}$ are each perpendicular to two of $\alpha, \beta, \gamma=-(\alpha * \beta)^{-1}$ and $\beta^{-1} * \alpha * \beta$ (see figure $\mathbb{1}$ ) is a fundamental domain for the $Y$ piece.

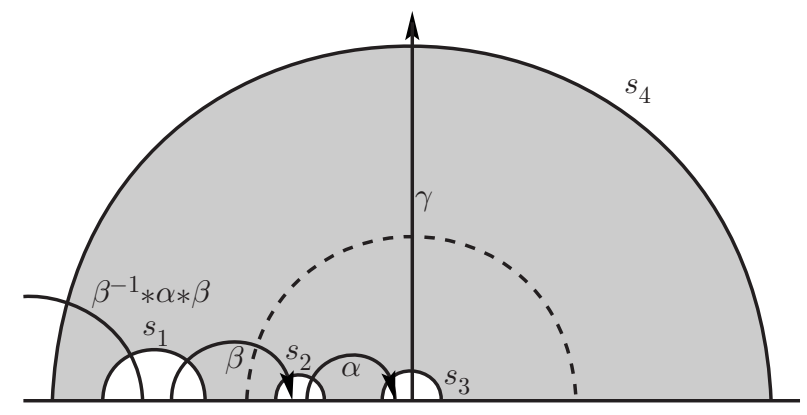

Figure 1. A fundamental domain of a $\mathrm{Y}$ piece

Proof. Using the side pairing transformations $g_{s_{1}}=h_{\beta}, g_{s_{2}}=h_{\beta}^{-1}, g_{s_{3}}=h_{\gamma}$ and $g_{s_{4}}=h_{\gamma}^{-1}$ we verify the conditions of Poincaré's polyhedron theorem (as presented in [Mas88]). Thus $\mathbb{H} /\left\langle h_{\beta}, h_{\gamma}\right\rangle=\mathbb{H} /\left\langle h_{\alpha}, h_{\beta}\right\rangle$ is a surface and its signature is $(0,3)$ as can easily be seen from the fundamental domain.

Remark. Note that a $\mathrm{Y}$ piece is not a compact surface and that the graphics of the $\mathrm{Y}$ and $\mathrm{X}$ pieces in this paper visualize only the Nielsen kernels (half-cylinders dropped).

\section{THE X PIECE}

In this section, we will study another surface which we will call the $\mathrm{X}$ piece. It's a Riemann surface of signature $(0,4)$ obtained by joining two $\mathrm{Y}$ pieces generated by $h_{\alpha}, h_{\beta}$ and $h_{\delta}, h_{\varepsilon}$ along $\gamma=-(\alpha * \beta)^{-1}=-\delta * \varepsilon$ (dropping two half-cylinders).

Using again Poincaré's polyhedron theorem, it is easy to prove that the fundamental domain of the $\mathrm{X}$ piece can be obtained by joining the domains of the two $\mathrm{Y}$ pieces along $\gamma$ as in figure 2 .

4.1. The coordinates in the quaternion basis. Obviously, if we take the hyperbolic elements $\alpha, \beta, \delta, \varepsilon$ such that $\alpha * \beta * \delta * \varepsilon=1$, we cannot be sure that the axes of $\alpha$ and $\varepsilon$ are situated on two different sides of $\gamma$, i.e. we cannot be sure that $\gamma$ is a dividing geodesic. In order to ensure this, we conjugate all the elements such that $\gamma=\left(c+\sqrt{c^{2}-1} \mathbf{I}\right)$, and the condition for $\gamma$ being a dividing geodesic is now equivalent to the condition that the attracting fixed points of $\alpha$ and $\varepsilon$ are of different signs (they are real numbers!); which leads us to proposition 10 .

Proposition 10. Let $\alpha, \beta, \gamma, \delta$ and $\varepsilon$ be hyperbolic elements such that $h_{\alpha}, h_{\beta}$ and $h_{\delta}, h_{\varepsilon}$ generate $Y$ pieces and $\gamma=-(\alpha * \beta)^{-1}=-\delta * \varepsilon=\left(c+\sqrt{c^{2}-1} \mathbf{I}\right)$. Suppose that the attracting fixed point of $\alpha$ and the repulsive fixed point of $\varepsilon$ are $-L \in \hat{\mathbb{R}}$ 


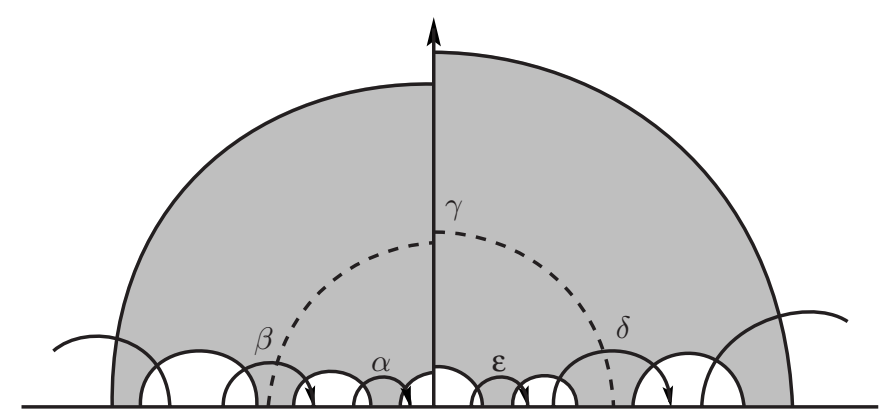

Figure 2. A fundamental domain of an $\mathrm{X}$ piece

and $M \in \hat{\mathbb{R}}$, then

$$
\begin{aligned}
& (a+A)=a-\frac{a c+b}{\sqrt{c^{2}-1}} \mathbf{I}-\frac{\tilde{L}}{2\left(c^{2}-1\right)}(\mathbf{J}+\mathbf{K})+\frac{h(a, b, c)}{2 \tilde{L}}(\mathbf{J}-\mathbf{K}) \\
& (b+B)=b-\frac{b c+a}{\sqrt{c^{2}-1}} \mathbf{I}-\frac{\tilde{L}\left(c+\sqrt{c^{2}-1}\right)}{2\left(c^{2}-1\right)}(\mathbf{J}+\mathbf{K})+\frac{h(a, b, c)\left(c-\sqrt{c^{2}-1}\right)}{2 \tilde{L}}(\mathbf{J}-\mathbf{K}), \\
& (d+D)=d+\frac{c d+e}{\sqrt{c^{2}-1}} \mathbf{I}-\frac{\tilde{M}\left(c+\sqrt{c^{2}-1}\right)}{2\left(c^{2}-1\right)}(\mathbf{J}+\mathbf{K})+\frac{h(c, d, e)\left(c-\sqrt{c^{2}-1}\right)}{2 \tilde{M}}(\mathbf{J}-\mathbf{K}), \\
& (e+E)=e+\frac{c e+d}{\sqrt{c^{2}-1}} \mathbf{I}-\frac{\tilde{M}}{2\left(c^{2}-1\right)}(\mathbf{J}+\mathbf{K})+\frac{h(c, d, e)}{2 \tilde{M}}(\mathbf{J}-\mathbf{K})
\end{aligned}
$$

where

$$
\begin{aligned}
\tilde{L} & =L \sqrt{c^{2}-1}\left(b+a c+\sqrt{a^{2}-1} \sqrt{c^{2}-1}\right), \\
\tilde{M} & =M \sqrt{c^{2}-1}\left(d+c e+\sqrt{c^{2}-1} \sqrt{e^{2}-1}\right), \\
h(u, v, w) & =u^{2}+v^{2}+w^{2}+2 u v w-1 .
\end{aligned}
$$

Proof. Using the special form of $\gamma=(c+C)=\left(c+\sqrt{c^{2}-1} \mathbf{I}\right)$ and the fact that $(A, C),(B, C),(D, C)$ and $(E, C)$ are known in terms of traces, we can calculate the components $a_{1}, b_{1}, d_{1}$ and $e_{1}$ (recall that the matrix without trace $A$ can be written as $\left.A=a_{1} \mathbf{I}+a_{2} \mathbf{J}+a_{3} \mathbf{K}\right)$.

We have the attracting fixed point of $\alpha\left(-L=\frac{a_{1}+\sqrt{a^{2}-1}}{a_{2}-a_{3}}\right)$ and the repulsive fixed point of $\varepsilon\left(M=\frac{e_{1}-\sqrt{e^{2}-1}}{e_{2}-e_{3}}\right)$. Thus we get $\left(a_{2}-a_{3}\right)$ and $\left(e_{2}-e_{3}\right)$. We know also that $\alpha$ and $\varepsilon$ are normalized, i.e. $a^{2}-1=a_{1}^{2}+\left(a_{2}-a_{3}\right)\left(a_{2}+a_{3}\right)$ and $e^{2}-1=$ $e_{1}^{2}+\left(e_{2}-e_{3}\right)\left(e_{2}+e_{3}\right)$. Therefore $\left(a_{2}+a_{3}\right)$ and $\left(e_{2}+e_{3}\right)$ can be expressed in terms of traces. The solution of this set of linear equations is (after simplification) $a_{2}, a_{3}$, $e_{2}$ and $e_{3}$.

We know that $(A, B)=-a b-c=a_{1} b_{1}+a_{2} b_{2}-a_{3} b_{3}$ and $(D, E)=-c-d e=$ $d_{1} e_{1}+d_{2} e_{2}-d_{3} e_{3}$. Thus we get $b_{2}$ and $d_{2}$ as functions of $b_{3}$ and $d_{3}$. As $\beta$ and $\delta$ are normalized $\left(b^{2}-1=b_{1}^{2}+b_{2}^{2}-b_{3}^{2}\right.$ and $\left.d^{2}-1=d_{1}^{2}+d_{2}^{2}-d_{3}^{2}\right)$ we can replace 
$b_{2}$ and $d_{2}$ by their functions of $b_{3}$ and $d_{3}$ in the normalizing condition. We get a quadratic equation for each of $b_{3}$ and $d_{3}$ and we can exclude one of the solutions for each of $b_{3}$ and $d_{3}$ because $b_{2}-b_{3}>0$ as well as $d_{2}-d_{3}>0$.

After simplification, we get the result.

Remark. This proposition, together with proposition 9 proves also that every $\mathrm{Y}$ piece can be constructed by a quotient $\mathbb{H} /\left\langle h_{\alpha}, h_{\beta}\right\rangle$, where $\alpha$ and $\beta$ satisfy $H_{Y}$ because we can choose $\tilde{L}=1$ by a simultaneous conjugation of $\alpha, \beta$ and $\gamma$.

4.2. The Teichmüller space. We have now expressed $\alpha, \beta, \gamma, \delta$ and $\varepsilon$ in terms of the traces $a, b, c, d$ and $e$ as well as the real numbers $\tilde{M}$ and $\tilde{L}$. Clearly, $a, b, c, d$ and $e$ are invariant to conjugation, but $\tilde{M}$ and $\tilde{L}$ aren't. We therefore introduce the elements $\xi=(x+X)=-(\alpha * \delta)^{-1}$ and $\zeta=(z+Z)=-(\beta * \delta)^{-1}$ that give us a certain measure of $\tilde{M}$ and $\tilde{L}$, and lead to the parameter space of the X piece in terms of traces only:

$$
\mathcal{P}=\left\{(a, b, c, d, e, x, z) \in \mathbb{R}^{7} \mid a, b, c, d, e, x, z>1\right\} .
$$

In fact we shall express $\tilde{M}$ and $\tilde{L}$ in terms of these traces if we really want $\mathcal{P}$ to be the parameter space. But before that, let us consider the following lemma as well as its corollary:

Lemma 11 (Helling). If $H$ is the following matrix of internal products,

$$
H=\left(\begin{array}{llll}
(A, A) & (A, B) & (A, C) & (A, D) \\
(B, A) & (B, B) & (B, C) & (B, D) \\
(C, A) & (C, B) & (C, C) & (C, D) \\
(D, A) & (D, B) & (D, C) & (D, D)
\end{array}\right)
$$

then $\operatorname{det}(H)=0$.

Proof. The dimension of 2 by 2 matrices without trace is 3 (they are generated by $\mathbf{I}, \mathbf{J}$ and $\mathbf{K})$. Therefore $A, B, C$ and $D$ are linearly dependent. Thus, the rows of $H$ are linearly dependent and $\operatorname{det}(H)=0$.

Remark. H. Helling gives this lemma in Hel74 in the wider context of eight elements $\left\{\alpha_{1}, \ldots, \alpha_{4}, \beta_{1}, \ldots, \beta_{4}\right\}$ of an abstract group $\Gamma$ and a trace function $s: H_{s}\left(\alpha_{1}, \ldots, \alpha_{4} ; \beta_{1}, \ldots, \beta_{4}\right)=\left(h_{i j}\right)_{1 \leq i, j \leq 4} ; h_{i j}=s\left(\alpha_{i} * \beta_{j}\right)-s\left(\alpha_{i}^{-1} * \beta_{j}\right)$. For $s=2 t r$, it is easy to see that $H=\frac{1}{4} H_{s}(\alpha, \beta, \gamma, \delta ; \alpha, \beta, \gamma, \delta)$.

Corollary 12. We have

$$
\begin{aligned}
Q= & a^{2}+b^{2}+c^{2}+d^{2}+e^{2}+x^{2}+z^{2}+4 a b d e-1 \\
& +2 c(a b+d e)+2 x(a d+b e)+2 z(a e+b d)-2 c x z=0 .
\end{aligned}
$$

Proof. Using the fact that $\alpha, \delta, \xi$ and $\beta, \delta, \zeta$ constitute $\mathrm{Y}$ pieces, we get $\operatorname{det}(H)=$ $\left(a^{2}+b^{2}+c^{2}+2 a b c-1\right) Q$. But we know that $a^{2}+b^{2}+c^{2}+2 a b c-1>0$, which gives us the result.

Proposition 13. The hyperbolic elements $(a+A),(b+B),(d+D)$ and $(e+E)$ with the coordinates of proposition 10 constitute an $X$ piece corresponding to 


$$
\begin{aligned}
& (a, b, c, d, e, x, z) \in \mathcal{P}, \text { iff } \\
& \quad \frac{\tilde{M}}{\tilde{L}}=-\frac{a e+b d+(a d+b e)\left(c-\sqrt{c^{2}-1}\right)+\sqrt{c^{2}-1}\left(\left(c-\sqrt{c^{2}-1}\right) z-x\right)}{h(a, b, c)} .
\end{aligned}
$$

Let's call the right-hand expression $p$.

Proof. We must show that $(a+A),(b+B),(d+D)$ and $(e+E)$ corresponding to $(a, b, c, d, e, x, z) \in \mathcal{P}$ are such that

$$
(A, D)=-a d-x \quad \text { and } \quad(B, D)=-b d-z .
$$

Furthermore we have to prove that $p>0$ which implies a situation as in figure 2 (fixed points of $\alpha$ and $\varepsilon$ of different signs).

With the coordinates of proposition [10, the equations $\dagger$ yield a non-degenerated system of two equations linear in $\tilde{p}=\frac{\tilde{M}}{\tilde{L}}$ and $\tilde{q}=\frac{\tilde{L}}{\tilde{M}}$. The unique solution for $\tilde{p}$ is $p$ but the solution for $\tilde{q}$ is not its inverse at first sight. However, we can extract a factor $Q$ from $\tilde{p} \tilde{q}-1$ which is therefore zero because of corollary 12 .

The condition $p>0$ is clearly equivalent to

$$
a e+b d+(a d+b e)\left(c-\sqrt{c^{2}-1}\right)+\sqrt{c^{2}-1}\left(\left(c-\sqrt{c^{2}-1}\right) z-x\right)<0 .
$$

If we solve this condition for $z$, we get $z<\frac{\sqrt{c^{2}-1} x-(a e+b d)-(a d+b e)\left(c-\sqrt{c^{2}-1}\right)}{\sqrt{c^{2}-1}\left(c-\sqrt{c^{2}-1}\right)}$. Let's call this last expression $z_{\text {asym }}$.

Let's now consider the polynomial of the corollary 12 as a function $Q(x, z)$ whose zero-set is a hyperbola. The branch of this hyperbola with $x$ and $z$ positive has an upper asymptote (with respect to $z$ ) given by the equation $z=z_{\text {asym. Thus }}$ $z<z_{\text {asym }}$ for all $(x, z)$ on the branch of the hyperbola. This means $p>0$ for any set of parameters satisfying $\operatorname{det}(H)=0$.

Remark. If we want to fix the fundamental domain of the $\mathrm{X}$ piece (i.e. not only up to a homothetic transformation) we can for instance fix $\tilde{M}=p$ and $\tilde{L}=1$ in the formula of proposition 10 .

Theorem A. The subgroup of $S L(2, \mathbb{R})$ acting on the upper half plane $\mathbb{H}$ by Möbius transformations such that the quotient surface has signature $(0,4)$, is freely generated by $(a+A),(b+B)$ and $(d+D)$ or a simultaneous conjugation of these three; where $(a+A),(b+B)$ and $(d+D)$ are the ones given in proposition 10 , $\tilde{M}=-\frac{a e+b d+(a d+b e)\left(c-\sqrt{c^{2}-1}\right)+\sqrt{c^{2}-1}\left(\left(c-\sqrt{c^{2}-1}\right) z-x\right)}{h(a, b, c)}$ and $\tilde{L}=1$.

Where $(a, b, c, d, e, x, z)$ is an element of Teichmüller space $\mathcal{T}$ of surfaces of signature $(0,4)$ in trace coordinates, i.e.

$$
\mathcal{T}:=\{(a, b, c, d, e, x, z) \in \mathcal{P} \mid Q=0\}
$$

with the parameter space

$$
\mathcal{P}:=\left\{(a, b, c, d, e, x, z) \in \mathbb{R}^{7} \mid a, b, c, d, e, x, z>1\right\}
$$

and the polynomial

$$
\begin{aligned}
Q:= & a^{2}+b^{2}+c^{2}+d^{2}+e^{2}+x^{2}+z^{2}+4 a b d e-1 \\
& +2 c(a b+d e)+2 x(a d+b e)+2 z(a e+b d)-2 c x z=0 .
\end{aligned}
$$

Proof. Direct deduction from proposition 10 and proposition 13 together with Poincaré's polyhedron theorem showing that figure 2 is the fundamental domain for the $\mathrm{X}$ piece. 
Remark. If we know the generating elements $\alpha, \beta$ and $\delta$ of an $\mathrm{X}$ piece in terms of (positive trace) matrices, we can easily extract these trace coordinates of Teichmüller space:

$(a, b, c, d, e, x, z)=(\operatorname{tr}(\alpha), \operatorname{tr}(\beta),-\operatorname{tr}(\alpha * \beta), \operatorname{tr}(\delta), \operatorname{tr}(\alpha * \beta * \delta),-\operatorname{tr}(\alpha * \delta),-\operatorname{tr}(\beta * \delta))$.

On the other hand, given a point in Teichmüller space, we can calculate the matrices (up to conjugation) using proposition 10.

Note also that $\left\{(a, b, c, d, e, x, z) \in \mathbb{R}^{7} \mid Q=0\right\}$ contains Teichmüller spaces of other surfaces freely generated by three elements that are not necessarily hyperbolic and such that the geodesics corresponding to $\alpha, \beta$ and $\delta$ might intersect (such as triangle groups, twice punctured tori, etc.).

\section{THE MOdULAR GROUP}

It is possible to represent the same surface by different points in Teichmüller space. The transformations mapping one of these points to another form a group (see for instance [SS92]), the modular group.

The aim of this section is to prove the following theorem:

Theorem B. The modular group of Teichmüller space $\mathcal{T}$ of surfaces of signature $(0,4)$ acts polynomially on $\mathcal{T}$ in these trace coordinates.

5.1. The generating elements. In order to prove theorem B, we show that there are four polynomial elements of the modular group that generate the whole group. The fact that the inverses of these elements are also polynomial implies that the modular group contains only polynomial elements. But before giving the generating elements, we state a well-known fact concerning quadratic equations which we will use later on:

Lemma 14. Let $P(x) \in \mathbb{R}[x]$ be a polynomial of degree 2 with leading coefficient 1 and $P(1)>0$. If the equation $P(x)=0$ has a real solution $\lambda_{1}>1$, then its other solution $\lambda_{2}$ is also greater than 1 .

Proof. We know $P(x)=\left(x-\lambda_{1}\right)\left(x-\lambda_{2}\right), P(1)>0$ and $1-\lambda_{1}<0$. Thus $1-\lambda_{2}<0$.

Proposition 15. The following transformations are elements of the modular group of Teichmüller space:

$$
\begin{aligned}
\varphi_{Y_{a}} & : \mathcal{T} \longrightarrow \mathcal{T}:(a, b, c, d, e, x, z) \longmapsto(b, a, c, d, e, 2(c x-a e-b d)-z, x), \\
\varphi_{Y_{d}} & : \mathcal{T} \longrightarrow \mathcal{T}:(a, b, c, d, e, x, z) \longmapsto(a, b, c, e, d, 2(c x-a e-b d)-z, x), \\
\varphi_{\text {turn }} & : \mathcal{T} \longrightarrow \mathcal{T}:(a, b, c, d, e, x, z) \longmapsto(b, d, z, e, a, 2(c z-a d-b e)-x, c) .
\end{aligned}
$$

Proof. In each case we we look for elements $\alpha^{\prime}, \beta^{\prime}, \delta^{\prime}, \varepsilon^{\prime} \in S L(2, \mathbb{R})$ such that the group of isometries $\left\langle h_{\alpha}, h_{\beta}, h_{\delta}, h_{\varepsilon}\right\rangle$ is the same as $\left\langle h_{\alpha}^{\prime}, h_{\beta}^{\prime}, h_{\delta}^{\prime}, h_{\varepsilon}^{\prime}\right\rangle$ and such that $\alpha^{\prime} * \beta^{\prime} * \delta^{\prime} * \varepsilon^{\prime}=1$. Then we must show that $\varphi(a, b, c, d, e, x, z)=\left(\operatorname{tr}\left(\alpha^{\prime}\right), \operatorname{tr}\left(\beta^{\prime}\right)\right.$, $\left.-\operatorname{tr}\left(\alpha^{\prime} * \beta^{\prime}\right), \operatorname{tr}\left(\delta^{\prime}\right), \operatorname{tr}\left(\varepsilon^{\prime}\right),-\operatorname{tr}\left(\alpha^{\prime} * \delta^{\prime}\right),-\operatorname{tr}\left(\beta^{\prime} * \delta^{\prime}\right)\right)$ and that these coordinates are all greater than 1 . Thus they necessarily satisfy the condition $\operatorname{det}\left(H^{\prime}\right)=0$ because that is a consequence of $\operatorname{dim}\left(H_{0}\right)=3$.

In the first case we choose $\alpha^{\prime}=\alpha * \beta * \alpha^{-1}, \beta^{\prime}=\alpha, \delta^{\prime}=\delta, \varepsilon^{\prime}=\varepsilon$. They clearly generate the same group, $\alpha^{\prime} * \beta^{\prime} * \delta^{\prime} * \varepsilon^{\prime}=\alpha * \beta * \alpha^{-1} * \alpha * \delta * \varepsilon=1$ and calculating the traces of the new elements we get the coordinates of $\varphi_{Y_{a}}(a, b, c, d, e, x, z)$. Considering the polynomial of corollary 12 as a polynomial $Q(z)$ and using lemma 14 
we prove that the two solutions $z$ and $2(c x-a e-b d)-z$ to the equation $Q(z)=0$ are both greater than 1 . Thus the coordinates of $\varphi_{Y_{a}}(a, b, c, d, e, x, z)$ are all greater than 1.

In the second case we choose $\alpha^{\prime}=\alpha, \beta^{\prime}=\beta, \delta^{\prime}=\delta * \varepsilon * \delta^{-1}$ and $\varepsilon^{\prime}=\delta$.

In the third case we choose $\alpha^{\prime}=\beta, \beta^{\prime}=\delta, \delta^{\prime}=\varepsilon$ and $\varepsilon^{\prime}=\alpha$. Using lemma 14 for the polynomial equation $Q(x)=0$, we prove that the two solutions $x$ and $2(c z-a d-b e)$ $-x$ are both greater than 1 . Thus the coordinates of $\varphi_{t u r n}(a, b, c, d, e, x, z)$ are all greater than 1 as well.

Remark. The inverses of $\varphi_{Y_{a}}, \varphi_{Y_{d}}$ and $\varphi_{t u r n}$ are also polynomial:

$$
\begin{aligned}
\varphi_{Y_{a}}^{-1} & : \mathcal{T} \longrightarrow \mathcal{T}:(a, b, c, d, e, x, z) \longmapsto(b, a, c, d, e, z, 2(c z-a d-b e)-x), \\
\varphi_{Y_{d}}^{-1} & : \mathcal{T} \longrightarrow \mathcal{T}:(a, b, c, d, e, x, z) \longmapsto(a, b, c, e, d, z, 2(c z-a d-b e)-x), \\
\varphi_{\text {turn }}^{-1} & : \mathcal{T} \longrightarrow \mathcal{T}:(a, b, c, d, e, x, z) \longmapsto(e, a, z, b, d, 2(c z-a d-b e)-x, c) .
\end{aligned}
$$

Geometrically, an element of the modular group can be interpreted as a choice of other geodesics on the same surface. Figure 3 shows this for the transformations of proposition 15 .

As Teichmüller space can be defined as the space of isotopy classes of marked complex structures of the Riemann surface (where two structures define the same point in Teichmüller space if there exists a holomorphic homeomorphism homotopic to the identity leading from one to the other), we can interpret an element of the modular group as a homeomorphism between marked $\mathrm{X}$ pieces up to holomorphic homeomorphisms homotopic to the identity. One representative of $\varphi_{Y_{a}}$ is thus the following transformation of the $\mathrm{X}$ piece: We (pointwise) fix the $\mathrm{Y}$ piece with border geodesics (corresponding to) $\delta, \varepsilon$ and $\gamma$. We deform the Y piece with border $\alpha$, $\beta$ and $\gamma$ such that $\gamma$ is fixed pointwise and such that the lengths of $\alpha$ and $\beta$ are exchanged $\left(\operatorname{tr}\left(\alpha^{\prime}\right)=\operatorname{tr}(\beta), \operatorname{tr}\left(\beta^{\prime}\right)=\operatorname{tr}(\alpha)\right)$. Then we twist this Y piece along $\gamma^{-1}$ half way round, fixing $\gamma$ pointwise.

It is obvious that transformations $\varphi_{Y_{a}}, \varphi_{Y_{d}}$ and $\varphi_{\text {turn }}$ cannot generate the modular group because they don't permit a change of orientation for $\gamma$ without an exchange of the sets $\{a, b\}$ and $\{d, e\}$. We thus introduce $\varphi_{i n v}$ in proposition 16 .

Proposition 16. The following transformation is an element of the modular group of Teichmüller space:

$$
\varphi_{i n v}: \mathcal{T} \longrightarrow \mathcal{T}:(a, b, c, d, e, x, z) \longmapsto(b, a, c, e, d, 2(c z-a d-b e)-x, z) .
$$

It is an involution.

Proof. We choose $\alpha^{\prime}=\beta^{-1}, \beta^{\prime}=\alpha^{-1}, \delta^{\prime}=\varepsilon^{-1}$ and $\varepsilon^{\prime}=\delta^{-1}$. Again they generate the same group and $\alpha^{\prime} * \beta^{\prime} * \delta^{\prime} * \varepsilon^{\prime}=\beta^{-1} * \alpha^{-1} * \varepsilon^{-1} * \delta^{-1}=(-\gamma) *\left(-\gamma^{-1}\right)=1$.

Calculating the traces of the new elements we get the coordinates of $\varphi_{\text {inv }}(a, b, c, d, e, x, z)$ (after some simplifications using corollary 12). The traces are greater than 1 analogously to the proof of proposition 15.

Direct calculation leads to $\varphi_{i n v} \circ \varphi_{i n v}(a, b, c, d, e, x, z)=(a, b, c, d, e, x, z)$.

In order to prove that the elements $\varphi_{Y_{a}}, \varphi_{Y_{d}}, \varphi_{\text {turn }}$ and $\varphi_{\text {inv }}$ generate the whole modular group, we need a result on a subgroup of the modular group of $\mathrm{X}$ pieces fixing one of the building blocks ( $\mathrm{Y}$ pieces). 


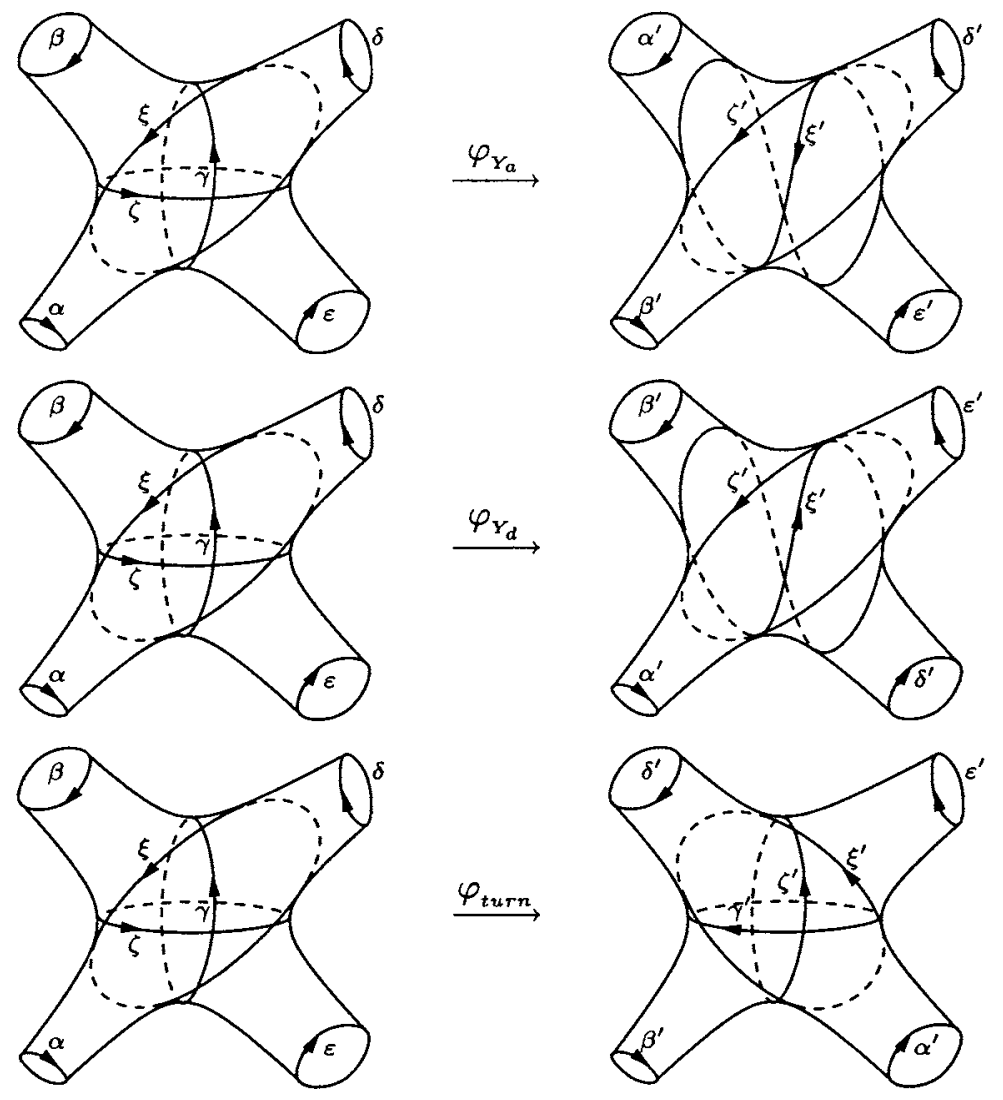

Figure 3. Other choices of the geodesics

Lemma 17. The set of elements of the modular group of the $X$ piece that have representatives that fix (pointwise) the $Y$ piece whose border geodesics correspond to $\delta$, $\varepsilon$ and $\gamma$ form a cyclic subgroup generated by $\varphi_{Y_{a}}$.

Proof. Let $\psi$ be a generic element of the set and consider $\tilde{\psi}$, its action on the generators $(\alpha, \beta, \delta, \varepsilon) \in S L(2, \mathbb{R})^{4}$. As the "right" Y piece (whose border geodesics correspond to $\delta, \varepsilon$ and $\gamma)$ is fixed, we can consider its action on $(\alpha, \beta) \in S L(2, \mathbb{R})^{2}$ only $\left(\delta\right.$ and $\varepsilon$ are fixed), i.e. $\tilde{\psi}: S L(2, \mathbb{R})^{2} \longrightarrow S L(2, \mathbb{R})^{2}:(\alpha, \beta) \longmapsto\left(\alpha^{\prime}, \beta^{\prime}\right)$ such that $\alpha * \beta=\alpha^{\prime} * \beta^{\prime}$.

It is easy to see that they form a group and it only remains to show that the action of $\varphi_{Y_{a}}$ defined by

$$
\tilde{\varphi}_{Y}:(\alpha, \beta) \longmapsto\left(\alpha * \beta * \alpha^{-1}, \alpha\right)
$$

generates the whole group.

As $\alpha * \beta=\tilde{\psi}(\alpha) * \tilde{\psi}(\beta)$, the action $\tilde{\psi}$ maps $(\alpha, \beta)$ to either one of the following:

(1) $\left(\alpha * \beta * g, g^{-1}\right)$,

(2) $\left(\alpha * g, g^{-1} * \beta\right)$ or

(3) $\left(g, g^{-1} * \alpha * \beta\right)$,

where $g$ is a length-reduced word in $\alpha$ and $\beta$. 
Note that $\alpha^{\prime}=\tilde{\psi}(\alpha)$ as well as $\tilde{\psi}(\beta)$ must be conjugates of $\alpha$ and $\beta$ because their corresponding geodesics together with the geodesic corresponding to $\gamma$ must form the "left" Y piece and thus $\{\operatorname{tr}(\alpha), \operatorname{tr}(\beta)\}=\left\{\operatorname{tr}\left(\alpha^{\prime}\right), \operatorname{tr}\left(\beta^{\prime}\right)\right\}$ and the geodesics corresponding to $\alpha^{\prime}$ and $\beta^{\prime}$ cannot change orientation (cf. proposition 8 , the orientation of the geodesic corresponding to $\gamma$ is fixed).

Let us first consider the second case: Either $g$ starts with $\alpha^{-1}$ and we are in case (3) or it ends with $\alpha^{-1}$ (because $\alpha * g$ must be a conjugate to $\alpha$ or $\beta$ ). But in that case $g^{-1}$ starts with $\alpha$ and therefore ends with $\beta^{-1}$ (because $g^{-1} * \beta$ is a conjugate to $\alpha$ or $\beta$ ) and we are in the case (1).

Let us now consider case (1) and prove the hypothesis by induction on the length of $g$ : If $g=\beta^{-1},(\alpha, \beta)$ maps to $(\alpha, \beta)$ and $\tilde{\psi}=i d$, if $g=\alpha^{-1},(\alpha, \beta)$ maps to $\left(\alpha * \beta * \alpha^{-1}, \alpha\right)$ and $\tilde{\psi}=\tilde{\varphi}_{Y}$. Other one-letter words are not possible because $\alpha * \beta * g$ as well as $g^{-1}$ must be conjugates to $\alpha$ or $\beta$. Suppose now that the length of $g$ is $n>1$ and that the hypothesis is true for any word strictly shorter than $n$. If $g$ doesn't begin with $\beta^{-1}$, then $\tilde{\varphi}_{Y}^{-1}\left(\alpha * \beta * g, g^{-1}\right)=\left(g^{-1}, g * \alpha * \beta * g * g^{-1}\right)=$ $\left(\alpha * \beta * g^{\prime}, g^{\prime-1}\right)$ with length of $g^{\prime}$ smaller than $n$ because $g$ must end with $\beta^{-1} * \alpha^{-1}$ and is a conjugate to $\alpha$ or $\beta$. If $g$ does begin with $\beta^{-1}$, then it must begin with $\beta^{-1} * \alpha^{-1}$ because $\alpha * \beta * g$ and $g^{-1}$ must both be conjugates to $\alpha$ or $\beta$. But in that case $\tilde{\varphi}_{Y}\left(\alpha * \beta * g, g^{-1}\right)=\left(\alpha * \beta * g * g^{-1} * g^{-1} * \beta^{-1} * \alpha^{-1}\right)=\left(\alpha * \beta * g^{\prime}, g^{\prime-1}\right)$ with length of $g^{\prime}$ smaller than $n$.

The proof of case (3) is analogous to the one of case (1).

Proposition 18. The elements $\varphi_{Y_{a}}, \varphi_{Y_{d}}, \varphi_{\text {turn }}$ and $\varphi_{\text {inv }}$ generate the modular group of the $X$ piece.

Proof. We have to prove that using these elements we can generate any set of equivalent matrices $\left\{\alpha^{\prime}, \beta^{\prime}, \delta^{\prime}, \varepsilon^{\prime}\right\}$.

By the previous lemma, we know that the subgroup of the modular group of the $\mathrm{X}$ piece that has representatives that fix $\gamma=\alpha * \beta$ as well as $\delta$ and $\varepsilon$ is generated by $\varphi_{Y_{a}}$; the one that has representatives that fix $\gamma=\alpha * \beta$ as well as $\alpha$ and $\beta$ is generated by $\varphi_{Y_{d}}$. Therefore, using $\varphi_{Y_{a}}, \varphi_{Y_{d}}$ and $\varphi_{i n v}$, we can generate any set of equivalent matrices $\left\{\alpha^{\prime}, \beta^{\prime}, \delta^{\prime}, \varepsilon^{\prime}\right\}$ such that the dividing geodesic corresponding to $\gamma$ is fixed (but not its direction).

It remains to prove that we can generate any dividing geodesic. This we will do in the following subsection.

Remark. The group of permutations $S_{4}$ acting on the set $\{a, b, d, e\}$ is a subgroup of the modular group of the X piece (and thus of the automorphism group of the polynomial $Q$ as studied in BG99]). In fact, the modular group can be written as an amalgamated product $S_{4} *_{G} \mathbb{Z}_{2}$ of $S_{4}$ and $\mathbb{Z}_{2}$ over some non-trivial group $G$, where $S_{4}$ is generated by $s_{1}=\varphi_{i n v} \circ \varphi_{Y_{d}}, s_{2}=\varphi_{Y_{d}}^{-1} \circ \varphi_{\text {turn }} \circ \varphi_{i n v} \circ \varphi_{Y_{d}}$ and $s_{3}=\varphi_{i n v} \circ \varphi_{Y_{a}}$ and $\mathbb{Z}_{2}$ by $\varphi_{i n v}$.

Proof. The modular group can be generated by $s_{1}, s_{2}, s_{3}$ and $\varphi_{i n v}$, because $\varphi_{Y_{a}}=$ $\varphi_{i n v} \circ s_{3}, \varphi_{Y_{d}}=\varphi_{i n v} \circ s_{1}$ and $\varphi_{\text {turn }}=\varphi_{i n v} \circ s_{1} \circ s_{2} \circ s_{1}$.

$s_{1}, s_{2}$ and $s_{3}$ generate $S_{4}$ with the representation (see for instance [Art47]),

$$
\left\langle s_{1}, s_{2}, s_{3} \mid s_{1}^{2}=s_{2}^{2}=s_{3}^{2}=1, s_{1} s_{2} s_{1}=s_{2} s_{1} s_{2}, s_{2} s_{3} s_{2}=s_{3} s_{2} s_{3}, s_{1} s_{3}=s_{3} s_{1}\right\rangle,
$$

because all these relations hold. 
$G$ is non-trivial because there are some relations between $\varphi_{i n v}$ and $s_{i}$, for instance $s_{1} \circ \varphi_{i n v} \circ s_{1}=s_{3} \circ \varphi_{i n v} \circ s_{3}$.

5.2. Dividing geodesics. In this subsection we will need some notions introduced in Sem88 as well as some new ones concerning dividing geodesics without selfintersections, dividing the $\mathrm{X}$ piece into two $\mathrm{Y}$ pieces:

Definition 19. A geodesic segment without self-intersection on a Y piece with endpoints on one or two boundary components will be called an ard 3 . Two nonintersecting arcs are said to be parallel or homotopic if there exists a homeomorphism of the $\mathrm{Y}$ piece leaving the boundary components invariant and transforming one arc into the other. A road is a simply connected domain on the Y piece between two adjacent parallel arcs. A square is a connected domain on the Y piece that is not a road (if it is delimited by one geodesic arc and completely contains a border geodesic, it will be called a roundabout). A long-road is a series of roads (alternating on one of the $\mathrm{Y}$ pieces) on the $\mathrm{X}$ piece (two $\mathrm{Y}$ pieces glued together along a border geodesic) leading from a roundabout to another.

In the following figure we illustrate these notions on an easy example:

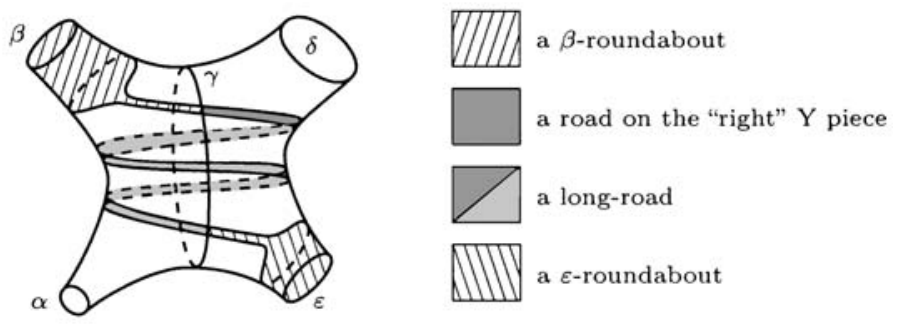

FiguRE 4. Roundabouts, roads and a long-road on an X piece

If $\eta$ is a dividing geodesic other than $\gamma$, then the $\mathrm{X}$ piece can be colored in red and white (say) such that the colours change along $\eta$.

Let's first consider only one $\mathrm{Y}$ piece defined by $\gamma$ and two appropriate border geodesics: As the $\mathrm{Y}$ piece is (topologically) nothing else but a one-holed cylinder (border of the hole $=\gamma$ ), there can only be one homotopy class for the arcs of $\eta$ (the endpoints must lie on $\gamma$ ). Thus $\eta$ delimits an $\alpha$-roundabout (colored red, say), a $\beta$-roundabout (can be either red or white) and some red and white roads in between.

If the $\alpha$ - and $\beta$-roundabout have the same color (red), the geodesic $\eta$ intersects $\gamma$ in a multiple of four points $(|\eta \cap \gamma| \bmod 4=0)$ otherwise $|\eta \cap \gamma| \bmod 4=2$.

Definition 20. A neighbor of a dividing geodesic $\mu$ is a dividing geodesic $\nu$ such that $|\mu \cap \nu|=4$.

\footnotetext{
${ }^{3}$ In this paper we only consider arcs with endpoints on the same boundary component.
} 
If the $\delta$-roundabout is red (this implies the $\beta$-roundabout is white), we apply $\varphi_{\text {turn }}^{-1} \circ \varphi_{Y_{a}}^{-1} \circ \varphi_{\text {turn }}$. If the $\varepsilon$-roundabout is red, we apply $\varphi_{\text {turn }}^{-1}$. In the new configuration, the new $\alpha$ - and $\beta$-roundabouts are red. In order to prove that we can generate any dividing geodesic, it is thus enough to prove that we can generate any dividing geodesic that doesn't divide $\alpha$ and $\beta$.

We can therefore assume that the $\alpha$ - and $\beta$-roundabouts are red. Thus there must be a red long-road connecting the $\alpha$ - and the $\beta$-roundabout. But the border of such a long-road together with the two roundabouts is a closed curve, thus all the arcs of $\eta$ are on this long-road.

To complete the proof of proposition 18 we have to show that any long-road $\eta$ connecting the $\alpha$ - to the $\beta$-roundabout is an iterated neighbor of $\gamma$ and that we can generate any direct neighbor. To do this, we introduce diagrams for long-roads leading from the $\alpha$ - to the $\beta$-roundabout corresponding to the geodesic $\eta$ :

The border of the $\beta$-roundabout is an arc $\hat{\eta}$ of $\eta$ and an $\operatorname{arc} \hat{\gamma}$ of $\gamma$. Following $\gamma$ (in its direction), we choose a point $P$ that is at a distance $\rho>0$ from $\hat{\gamma}$ such that there is no intersection of $\gamma$ and $\eta$ between $\hat{\gamma}$ and $P$. Now we punch a hole into the surface in $P$ and deform it into a disc in $\mathbb{R}^{2}$ (whose border corresponds to $P$ ) with four holes (that correspond to $\alpha, \beta, \delta$ and $\varepsilon$ ). We contract $\alpha, \beta, \delta$ and $\varepsilon$ to four points and deform the disc such that $\gamma$ is a vertical segment oriented upward and such that the points corresponding to $\alpha, \beta, \delta$ and $\varepsilon$ form a parallelogram whose side $(\alpha, \beta)$ is vertical. Not tracing the border of the disc corresponding to $P$, we get the diagram:

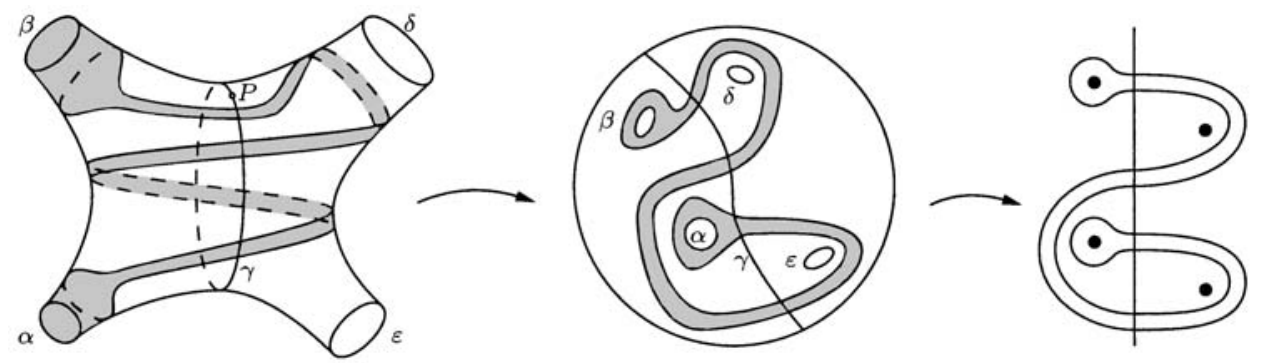

Figure 5. A long-road with its $\alpha$ - and $\beta$-roundabouts and its diagram

Remark. The choice of $P$ induces that there are no roads enclosing the $\beta$-roundabout in the diagram (such that an arc of the border of the road that is an arc of $\eta$ followed by an arc of $\gamma \backslash\{P\}$ is freely homotopic to $\beta$ ). But there may be several roads enclosing the $\alpha-, \delta$ - and $\varepsilon$-roundabouts.

If there are no roads enclosing the $\alpha$-roundabout, then $\eta$ is a neighbor of $\gamma$.

Two diagrams are equal if they there is a homeomorphism leading from one to the other. This means that a diagram of a dividing geodesic $\eta$ separating $\alpha$ and $\beta$ from $\delta$ and $\varepsilon$ is the same as the diagram of any multiple Dehn twist of $\eta$ along $\gamma$ or $\gamma^{-1}$

The following algorithm acting on diagrams will show that any long-road $\eta$ leading from the $\alpha$ - to the $\beta$-roundabout is an iterated neighbor of $\gamma$. In fact, at each step, it constructs a new long-road $\eta^{\prime}$ that is a neighbor of $\eta$ such that 
$|\eta \cap \gamma|>\left|\eta^{\prime} \cap \gamma\right|$; it stops when $\eta$ has been reduced to a neighbor of $\gamma$ :

\section{Algorithm.}

While the current diagram is not a neighbor of $\gamma$ do the $\alpha$-simplification step:

- orient the long-road from the $\alpha$ - towards the $\beta$-roundabout,

- with the road just beside the one leaving the $\alpha$-roundabout that is oriented in the same direction do the following transformation:

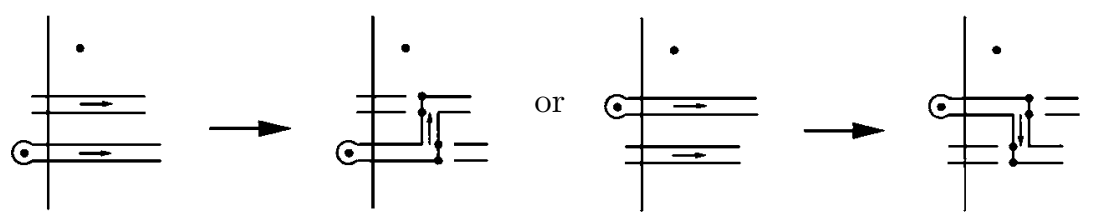

- remove the roads that are no longer part of the new long-road from the $\alpha$ to the $\beta$-roundabout;

Proof. At each simplification step we pass to a neighbor of the current diagram (the four intersection points are emphasised), thus the final diagram is an iterated neighbor of the original one. At each simplification step the number of parallel roads decreases strictly because some are left out.

The $\alpha$-simplification step is possible if there is a road that encloses the $\alpha$ roundabout as in figure 6 because in that case, this road can be oriented in either direction without prohibiting the simplification step.

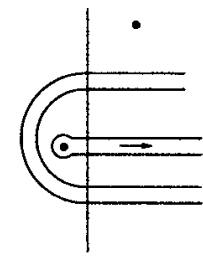

FiguRE $6 . \alpha$-simplification possible

Thus, the only situations where the $\alpha$-simplification step is not possible are the ones in figure 7 where $\eta$ is not a neighbor of $\gamma$ and the road leaving the $\alpha$-roundabout is either adjacent to the $\delta$ - or the $\varepsilon$-roundabout and the red road next to it has opposite direction, or there is no road enclosing the $\alpha$-roundabout.

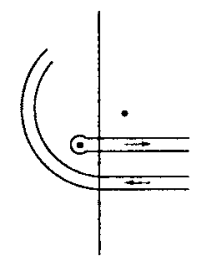

or

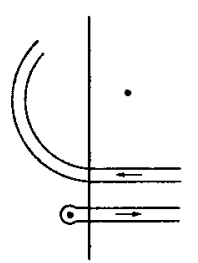

Figure 7. $\alpha$-simplification impossible

We will prove that these situations are impossible, finishing by that the proof of proposition 18 If the road leaving the $\alpha$-roundabout is either adjacent to the 
$\delta$ - or the $\varepsilon$-roundabout, then we must be in one of the situations of figure 8 and the $\alpha$-simplification is possible.

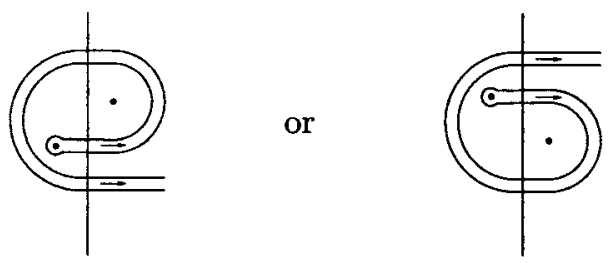

Figure 8. $\alpha$-simplification possible

If there is no road enclosing the $\alpha$-roundabout, $\eta$ must be a neighbor because of the choice of the diagrams.

This algorithm is not only an important step to the proof of proposition 18, it is also very useful to prove the following proposition:

Proposition 21. If the geodesic $\gamma$ is shorter than its neighbors, it is a shortest geodesic dividing the $X$ piece into two $Y$ pieces whose boundaries contain $\alpha, \beta$ and $\delta$, $\varepsilon$ respectively.

Proof. Let $\eta$ be any such dividing geodesic and $\gamma$ shorter than its neighbors. We have to prove that there is a length decreasing sequence of iterated neighbors leading from $\eta$ to $\gamma$. In fact, using the algorithm, we have already constructed this sequence. It remains to prove that the $\alpha$-simplification step is length decreasing.

Take the arc of $\eta$ that is part of the boundary of the $\alpha$-roundabout and give its intersection with $\gamma$ the names $A$ and $B$. Following $\gamma$ we get to the next intersections with $\eta$ and name them $C$ and $D$. We are thus in one of the following three situations:
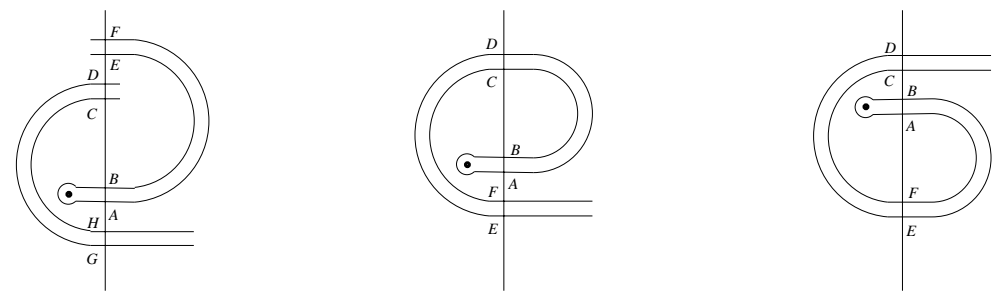

In the first situation the names $A B C D E F G H$ come in that order on $\gamma$ and in the order $A B E C H G D F$ on $\eta$. We can now construct the curves $\gamma^{\prime}$ and $\eta^{\prime}$, longer than and freely homotopic to neighbors of $\gamma$ and $n$ :

- For $\gamma^{\prime}$, we will first stay on $\eta$ from $A$ to $E$, then follow $\gamma^{-1}$ to $C, \eta$ to $H$, $\gamma^{-1}$ to $F$ and finish on $\eta$ to close the curve in $A$.

- For $\eta^{\prime}$, we follow $\gamma$ from $A$ to $C, \eta^{-1}$ to $E, \gamma$ to $F, \eta^{-1}$ to $H$ and finish on $\gamma$ to close the curve in $A$. Note that this curve is freely homotopic to the geodesic obtained by the $\alpha$-simplification step.

\footnotetext{
${ }^{4} \mathrm{~A}$ closed non-geodesic curve on any Riemann surface is strictly longer than its freely homotopic geodesic (see [FLP79]).
} 
It remains to prove that the length of $E$ to $F$ plus the length of $H$ to $C$ on $\gamma$ is strictly smaller than the length of $F$ to $E$ plus the length of $C$ to $H$ on $\eta$ : $l(E \stackrel{\gamma}{\longrightarrow} F)+l(H \stackrel{\gamma}{\longrightarrow} C)<l(F \stackrel{\eta}{\longrightarrow} E)+l(C \stackrel{\eta}{\longrightarrow} H)$. This implies that the length of $\eta^{\prime}$ is smaller than the length of $\eta$ (and thus that the $\alpha$-simplification step is length decreasing). Suppose now that $l(E \stackrel{\gamma}{\longrightarrow} F)+l(H \stackrel{\gamma}{\longrightarrow} C) \geq l(F \stackrel{\eta}{\longrightarrow} E)$ $+l(C \stackrel{\eta}{\longrightarrow} H)$. This implies $l\left(\gamma^{\prime}\right)-l(\eta) \leq l(\gamma)-l(\eta)$ and thus $l\left(\gamma^{\prime}\right) \leq l(\gamma)$ which is in contradiction to the hypothesis that $\gamma$ is shorter than its neighbors.

In the second situation the names $A B C D E F$ come in that order on $\gamma$ and in the order $A B C F E D$ on $\eta$. We can now construct the curves $\gamma^{\prime}$ and $\eta^{\prime}$ :

- For $\gamma^{\prime}$, we follow $\eta$ from $A$ to $F$, then follow $\gamma^{-1}$ to $D$ and finish on $\eta$ to close the curve in $A$.

- For $\eta^{\prime}$, we follow $\gamma$ from $A$ to $D, \eta^{-1}$ to $F$ and finish on $\gamma$ to close the curve in $A$. Note that this curve is freely homotopic to the geodesic obtained by the $\alpha$-simplification step.

Suppose that $l(F \stackrel{\gamma}{\longrightarrow} D) \geq l(D \stackrel{\eta}{\longrightarrow} F)$. This implies $l\left(\gamma^{\prime}\right)-l(\eta) \leq l(\gamma)-l(\eta)$ and thus $l\left(\gamma^{\prime}\right) \leq l(\gamma)$ which is in contradiction to the hypothesis that $\gamma$ is shorter than its neighbors. Thus $l(F \stackrel{\gamma}{\longrightarrow} D)<l(D \stackrel{\eta}{\longrightarrow} F)$ and therefore $l\left(\eta^{\prime}\right)<l(\eta)$.

The proof for the third situation is exactly analogous to the one for the second (mirror situation).

It remains to prove that we can generate any neighbor of $\gamma$ :

Proposition 22. The neighbors of the dividing geodesic $\gamma$ are of the form $\varphi_{X} \circ$ $\varphi_{Y_{d}}^{n}(\gamma)$ where $n \in \mathbb{Z}$ and

$$
\varphi_{X}:=\varphi_{t u r n}^{-1} \circ \varphi_{Y_{d}} \circ \varphi_{t u r n} \circ \varphi_{Y_{a}}^{-1} \circ \varphi_{t u r n} .
$$

Proof. There are only two possible diagrams for neighbors:
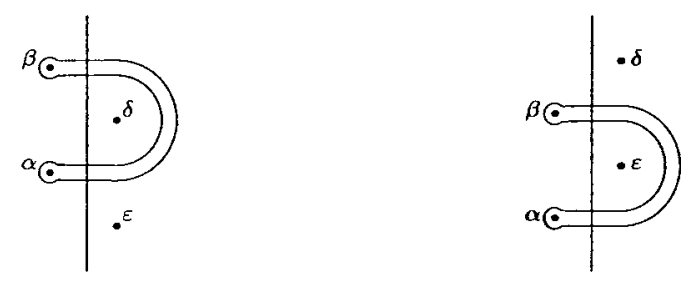

These two diagrams can be obtained by

$$
\varphi_{X}(\gamma)=-\delta^{-1} * \beta^{-1} * \delta * \alpha^{-1} \quad \text { and } \quad \varphi_{X} \circ \varphi_{Y_{d}}^{-1}(\gamma)=-\varepsilon^{-1} * \beta^{-1} * \varepsilon * \alpha^{-1}
$$

but each diagram corresponds to more than only one geodesic. Indeed, if two geodesics correspond to the same diagram there is a (multiple) Dehn twist along $\gamma$ that brings one onto the other. Therefore, all neighbors are of the form $-\gamma^{m} *$ $\delta^{-1} * \gamma^{-m} * \beta^{-1} * \gamma^{m} * \delta * \gamma^{-m} * \alpha^{-1}$ or $-\gamma^{m} * \varepsilon^{-1} * \gamma^{-m} * \beta^{-1} * \gamma^{m} * \varepsilon * \gamma^{-m} * \alpha^{-1}$ for some $m \in \mathbb{Z}$. But $\varphi_{X} \circ \varphi_{Y_{d}}^{2 m}(\gamma)=-\gamma^{m} * \varepsilon^{-1} * \gamma^{-m} * \beta^{-1} * \gamma^{m} * \varepsilon * \gamma^{-m} * \alpha^{-1}$ and $\varphi_{X} \circ \varphi_{Y_{d}}^{2 m-1}(\gamma)=-\gamma^{m} * \varepsilon^{-1} * \gamma^{-m} * \beta^{-1} * \gamma^{m} * \varepsilon * \gamma^{-m} * \alpha^{-1}$, hence the proposition. 


\section{A FUNDAMENTAL DOMAIN FOR THE MODULAR GROUP}

Now that we know the modular group, we can solve the Riemann moduli problem for surfaces of signature $(0,4)$ using the fundamental domain for the action of the modular group in Teichmüller space $\mathcal{T}$. This is the aim of this section.

We can get a pre-fundamental domain for the action of the modular group on $\mathcal{T}$ using the trace of $\gamma$ : For every element $\varphi$ of the modular group we want $\operatorname{tr}(\gamma) \leq$ $\operatorname{tr}(\varphi(\gamma))$. In order to get a fundamental domain we can thus proceed as follows:

Let $\gamma$ be a shortest dividing geodesic (equivalent to smallest possible trace) and orient it arbitrarily. It cuts the $\mathrm{X}$ piece into two $\mathrm{Y}$ pieces. Take the $\mathrm{Y}$ piece that contains the shortest boundary geodesic of the $\mathrm{X}$ piece, name this geodesic $\alpha$ and its third boundary geodesic $\beta$ and orient them such that $\alpha * \beta * \gamma=-1$. Let $\delta$ and $\varepsilon$ be the boundary geodesics of the X piece we haven't yet named and orient them such that $\alpha * \beta * \delta * \varepsilon=1$ (there are two possibilities to do this). Now we use $\varphi_{Y_{d}}$ or $\varphi_{Y_{d}}^{-1}$ repeatedly to "unwind" the $\mathrm{X}$ piece such that $x \leq c z-a d-b e$ and $z \leq c x-a e-b d$. By the following lemma 23, this situation gives a global minimum for $x+z$. To finish, we want to be in a unique situation and have $z \leq x$ which can be achieved by applying $\varphi_{i n v} \circ \varphi_{Y_{a}}: \mathcal{T} \longrightarrow \mathcal{T}:(a, b, c, d, e, x, z) \longmapsto(a, b, c, e, d, z, x)$ if needed. Actually, it is easy to prove that $z \leq x \leq c z-a d-b e$ and $\operatorname{det}(H)=0$ imply $z \leq c x-a e-b d$. Thus it only remains to translate "shortest dividing geodesic" into terms of traces in order to get a fundamental domain.

Lemma 23. Let $f: \mathcal{T} \rightarrow \mathbb{R}:(a, b, c, d, e, x, z) \mapsto x+z$ and $(a, b, c, d, e, x, z) \in \mathcal{T}$ such that $x \leq c z-a d-b e$ and $z \leq c x-a e-b d$. Then $f(a, b, c, d, e, x, z) \leq$ $f\left(\varphi_{Y_{d}}^{n}(a, b, c, \bar{d}, e, x, z)\right)$ for all $n \in \mathbb{Z}$.

Proof. $x \leq c z-a d-b e$ and $z \leq c x-a e-b d$ imply that $(a, b, c, d, e, x, z)$ is a local minimum of $f$ under the action of $\varphi_{Y_{d}}$. It remains to prove that it is also a global minimum, i.e. that $\hat{g}: \mathbb{Z} \rightarrow \mathbb{R}: n \mapsto f\left(\varphi_{Y_{d}}^{n}(a, b, c, d, e, x, z)\right)$ is a restriction of a convex function $g: \mathbb{R} \rightarrow \mathbb{R}$.

This is indeed the case as $\varphi_{Y_{d}}^{n}(a, b, c, d, e, x, z)=\left(a, b, c, d_{n}, e_{n}, x_{n}, z_{n}\right)$ can be written as

$$
\begin{aligned}
x_{n} & =\frac{1}{c^{2}-1}\left(\sqrt{h(a, b, c) h(c, d, e)} \cosh (A(n+1)+B)+k_{n+1}\right), \\
z_{n} & =\frac{1}{c^{2}-1}\left(\sqrt{h(a, b, c) h(c, d, e)} \cosh (A n+B)+k_{n}\right), \\
k_{n} & =c\left(a d_{n}+b e_{n}\right)+\left(a e_{n}+b d_{n}\right), \\
\left(d_{n}, e_{n}\right) & = \begin{cases}(d, e) & \text { if } n \text { even, } \\
(e, d) & \text { if } n \text { odd, }\end{cases}
\end{aligned}
$$

$$
\begin{aligned}
\text { where } \cosh (A) & =c \\
\cosh (B) & =\frac{z\left(c^{2}-1\right)-(c(a d+b e)+(a e+b d))}{\sqrt{h(a, b, c) h(c, d, e)}}, \\
\text { and } h(u, v, w) & =u^{2}+v^{2}+w^{2}+2 u v w-1
\end{aligned}
$$

This implies that $g$ can be given as

$$
t \mapsto \frac{\sqrt{h(a, b, c) h(c, d, e)}(\cosh (A(t+1)+B)+\cosh (A t+B))}{c^{2}-1}+\frac{(a+b)(d+e)}{c-1},
$$

which is a convex function in $t$. 
Remark. In [KR78] and [FR95], the authors investigate similar cases for two generator Fuchsian groups, showing the existence of a global minimum without explicitly giving a convex function.

Proposition 24. Let $\alpha=(a+A), \beta=(b+B), \delta=(d+D)$ and $\varepsilon=(e+E)$ be the generators of an $X$ piece, such that $a=\min \{a, b, d, e\}, z \leq x \leq c z-a d-b e$. Then $\gamma=-\delta * \varepsilon=(c+C)$ is a shortest dividing geodesic, iff $c \leq z$.

Proof. We must show that $\gamma$ is shorter than its iterated neighbors, $\xi, \zeta$ and their iterated neighbors (see section 5.2). We know that a dividing geodesic is shorter than its iterated neighbors if it is shorter than its neighbors (see proposition 21).

We show that $\bar{\gamma}:=\varphi_{X}(\gamma)$ is the shortest neighbor of $\gamma$ : Its trace is $2(x z-a b-d e)-c$. All other neighbors are of the form $\varphi_{X} \circ \varphi_{Y_{d}}^{n}(\gamma)$ (see proposition 22) and thus have traces $2\left(\operatorname{tr}\left(\varphi_{Y_{d}}^{n}(\xi)\right) \operatorname{tr}\left(\varphi_{Y_{d}}^{n}(\zeta)\right)-a b-d e\right)-c$. But as the $\mathrm{X}$ piece is unwound, $\operatorname{tr}\left(\varphi_{Y_{d}}^{n}(\xi)\right) \geq x$ and $\operatorname{tr}\left(\varphi_{Y_{d}}^{n}(\zeta)\right) \geq z$ for $n$ even and $\operatorname{tr}\left(\varphi_{Y_{d}}^{n}(\xi)\right) \geq z$ and $\operatorname{tr}\left(\varphi_{Y_{d}}^{n}(\zeta)\right) \geq x$ for $n$ odd.

We prove that $\gamma$ is shorter than its shortest neighbor $\bar{\gamma}$ if $c \leq z$. For this we construct two curves $\zeta_{1}$ and $\zeta_{2}$ both homotopic to $\zeta$ using only arcs of $\gamma$ and $\bar{\gamma}$ :

- For $\zeta_{1}$, we follow $\gamma$ from $A$ to $B$, then take $\bar{\gamma}$ to $C$, continue on $\gamma$ back to $B$ and finish on $\bar{\gamma}$ from $B$ to $A$.

- For $\zeta_{2}$, we follow $\gamma$ from $C$ to $D$, then take $\bar{\gamma}$ to $A$, continue on $\gamma$ back to $D$ and finish on $\bar{\gamma}$ from $D$ to $C$;

where $A, B, C, D$ are the intersections of $\gamma$ and $\bar{\gamma}$ as in figure 9 .

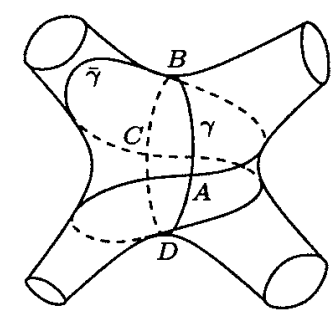

FiguRE 9. $\gamma$ and its neighbor $\bar{\gamma}=\varphi_{X}(\gamma)$

Using the argument that the geodesic is always the shortest curve of its homotopy class (see [FLP79]), we show that $\zeta$ is shorter than $\bar{\gamma}$ which implies that $\gamma$ is shorter than $\bar{\gamma}: l(\gamma)+l(\bar{\gamma})=l\left(\zeta_{1}\right)+l\left(\zeta_{2}\right)>2 l(\zeta)$. But $c \leq z$, therefore $l(\gamma) \leq l(\zeta)$, thus $l(\zeta)<l(\bar{\gamma})$ and finally $l(\gamma)<l(\bar{\gamma})$.

We show that $\zeta$ is shorter than its neighbors if $c \leq 2(x z-a b-d e)-c=\operatorname{tr}(\bar{\gamma})$ : Analogously to proposition [22, we can write the neighbors of $\zeta$ as $\varphi_{\text {turn }} \circ \varphi_{X} \circ$ $\varphi_{Y_{d}}^{n} \circ \varphi_{\text {turn }}^{-1}(\zeta)$ and show that the geodesic $\varphi_{\text {turn }} \circ \varphi_{X} \circ \varphi_{Y_{d}}^{-1} \circ \varphi_{\text {turn }}^{-1}(\zeta)$ is a shortest neighbor of $\zeta$ if $c \leq 2(x z-a b-d e)-c$ and $x \leq 2(c z-a d-b e)-x$. But $\operatorname{tr}\left(\varphi_{\text {turn }} \circ \varphi_{X} \circ \varphi_{Y_{d}}^{-1} \circ \varphi_{\text {turn }}^{-1}(\zeta)\right)=2(c x-a e-b d)-z \geq z$.

The proof that $\xi$ is shorter than its neighbors if $c \leq 2(x z-a b-d e)-c$ is analogous, using the following shortest neighbor of $\xi: \varphi_{Y_{a}}^{-1} \circ \varphi_{\text {inv }} \circ \varphi_{\text {turn }} \circ \varphi_{X} \circ \varphi_{Y_{d}}^{-1} \circ$ $\varphi_{\text {turn }}^{-1} \circ \varphi_{\text {inv }} \circ \varphi_{Y_{a}}(\xi)$ with trace $2(c z-a d-b e)-x \geq x$. 
This leads us to the fundamental domain $\mathcal{F}$ for the action of the modular group on Teichmüller space $\mathcal{T}$ that can be formulated as follows:

Theorem C. The set of isometry classes of surfaces of signature $(0,4)$ is in a 1-1-correspondence with the set

$$
\begin{aligned}
\mathcal{F}:=\left\{(a, b, c, d, e, x, z) \in \mathbb{R}^{7} \mid \begin{array}{l}
1<a \leq \min \{b, d, e\} \\
1<c \leq z \leq x \leq c z-a d-b e
\end{array}\right. \\
\text { and } Q=0\}
\end{aligned}
$$

where $Q$ is the following polynomial:

$$
\begin{aligned}
Q:= & a^{2}+b^{2}+c^{2}+d^{2}+e^{2}+x^{2}+z^{2}+4 a b d e-1 \\
& +2 c(a b+d e)+2 x(a d+b e)+2 z(a e+b d)-2 c x z=0 .
\end{aligned}
$$

Proof. Direct deduction from proposition 18 and proposition 24together with theorem A.

Remark. The set $\mathcal{F}$ is contractible.

\section{Fenchel-Nielsen COORdinates}

In this section we recall the definition of the Fenchel-Nielsen coordinates for Riemann surfaces (see for instance [SS92]) and try to find the generating elements of the modular group in these coordinates.

Let $\Sigma$ be a purely hyperbolic Riemann surface of signature $(g, b)$ and $\alpha_{1}, \alpha_{2}, \ldots$, $\alpha_{3 g-3+2 b}$ the oriented decomposing geodesics of a pants decomposition of $\Sigma$. On each $\alpha_{j}$ that is not a border geodesic, there exist two distinguished points $P_{j}^{1}$ and $P_{j}^{2}$ obtained as follows (some choices are required): Build the perpendiculars to $\alpha_{j}$ and one of the adjacent boundaries on each pair of pants (Y piece). They have $P_{j}^{1}$ and $P_{j}^{2}$ as common points with $\alpha_{j}$. Let $s_{j}$ denote the distance from $P_{j}^{1}$ to $P_{j}^{2}$ measured to the positive direction of $\alpha \sqrt{5}$. We can now define the Fenchel-Nielsen coordinates $l_{j}$ for $j=1,2, \ldots, 3 g-3+2 b$ and $\theta_{j}$ for $j$ such that $\alpha_{j}$ that is not a border geodesic, setting:

$$
\begin{aligned}
l_{j} & =\text { length of } \alpha_{j}, \\
\theta_{j} & =2 \pi s_{j} / l_{j} .
\end{aligned}
$$

In our case of an $\mathrm{X}$ piece we have thus the coordinates

$$
\left(l_{1}, l_{2}, l_{3}, l_{4}, l_{5}, \theta_{3}\right)=\left(l_{1}, l_{2}, l_{3}, l_{4}, l_{5}, \theta\right),
$$

corresponding to the geodesic lengths and a twisting angle as in figure 10

We can easily convert our trace coordinates into Fenchel-Nielsen coordinates but it is more difficult to do the opposite as we will see in the following two propositions.

Proposition 25. If the trace coordinates of an $X$ piece are $(a, b, c, d, e, x, z)$, then the Fenchel-Nielsen coordinates are

$$
\begin{aligned}
\left(l_{1}, l_{2}, l_{3}, l_{4}, l_{5}, \theta\right)= & \Psi(a, b, c, d, e, x, z) \\
= & (2 \operatorname{arccosh}(a), 2 \operatorname{arccosh}(b), 2 \operatorname{arccosh}(c), \\
& 2 \operatorname{arccosh}(d), 2 \operatorname{arccosh}(e), \theta),
\end{aligned}
$$

\footnotetext{
${ }^{5}$ If we want the coordinates of Teichmüller space (and not already the quotient of it by some elements of the modular group), we have to admit also distances $s_{j}$ greater than $l_{j}$ (going around $\alpha_{j}$ more than once) and even negative ones (following the opposite direction of $\alpha_{j}$ ); i.e. $s_{j} \in \mathbb{R}$.
} 


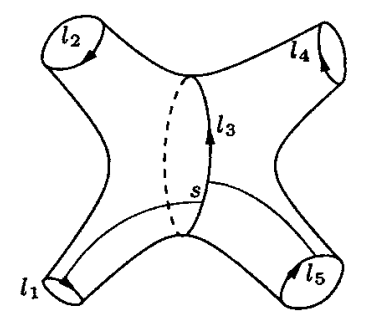

FiguRE 10. Fenchel-Nielsen coordinates

where

$$
\theta=2 \pi \frac{\ln (p(a, b, c, d, e, x, z) h(a, b, c))-\ln (h(c, d, e))}{4 \operatorname{arccosh}(c)}
$$

with

and

$$
h(u, v, w)=u^{2}+v^{2}+w^{2}+2 u v w-1
$$

$$
p(a, b, c, d, e, x, z)=-\frac{a e+b d+(a d+b e)\left(c-\sqrt{c^{2}-1}\right)+\sqrt{c^{2}-1}\left(\left(c-\sqrt{c^{2}-1}\right) z-x\right)}{h(a, b, c)} .
$$

Proof. The trace of a closed geodesic is the hyperbolic cosine of half of its length (see Sem88]). It thus only remains to construct $\theta$. In order to get the distinguished points on $\gamma$ we build the common perpendicular $\pi_{\gamma, \alpha}$ to $\gamma$ and $\alpha\left(\pi_{\gamma, \alpha}:=(\sqrt{1+(C \wedge A, C \wedge A)}+C \wedge A)\right)$ and the common perpendicular $\pi_{\gamma, \varepsilon}$ to $\gamma$ and $\varepsilon$ and intersect them with $\gamma$ (these intersection points correspond to $\pi_{\gamma, \pi_{\gamma, \alpha}}$ and $\left.\pi_{\gamma, \pi_{\gamma, \varepsilon}}\right)$. Using proposition 10 and definition 3 we get the points $\sqrt{|\tilde{L}| /\left(\left(c^{2}-1\right) h(a, b, c)\right)} i$ and $\sqrt{|\tilde{M}| /\left(\left(c^{2}-1\right) h(c, d, e)\right)} i$ in the upper half plane. Thus their (signed) distance is

$$
s=\frac{1}{2}(\ln (p(a, b, c, d, e, x, z) h(a, b, c))-\ln (h(c, d, e))) .
$$

This determines $\theta$.

Proposition 26. If we know that the Fenchel-Nielsen coordinates of an $X$ piece are $\left(l_{1}, l_{2}, l_{3}, l_{4}, l_{5}, \theta\right)$, then the trace coordinates are

$$
\begin{aligned}
(a, b, c, d, e, x, z) & =\Phi\left(l_{1}, l_{2}, l_{3}, l_{4}, l_{5}, \theta\right) \\
& =\left(\cosh \left(\frac{l_{1}}{2}\right), \cosh \left(\frac{l_{2}}{2}\right), \cosh \left(\frac{l_{3}}{2}\right), \cosh \left(\frac{l_{4}}{2}\right), \cosh \left(\frac{l_{5}}{2}\right), x, z\right)
\end{aligned}
$$

where $(x, z)$ is the solution of the following system of equations:

$$
\begin{cases}Q(a, b, c, d, e, x, z) & =0, \\ e^{2 s} \frac{h(c, d, e)}{h(a, b, c)} & =p(a, b, c, d, e, x, z),\end{cases}
$$

with

$$
\begin{array}{lll}
Q(a, b, c, d, e, x, z) & =a^{2}+b^{2}+c^{2}+d^{2}+e^{2}+x^{2}+z^{2}+4 a b d e-1 \\
& \quad+2 c(a b+d e)+2 x(a d+b e)+2 z(a e+b d)-2 c x z \\
s & =\frac{\theta}{2 \pi} l_{3}, \\
h(u, v, w) & =u^{2}+v^{2}+w^{2}+2 u v w-1 \quad \text { and } \\
p(a, b, c, d, e, x, z)= & -\frac{a e+b d+(a d+b e)\left(c-\sqrt{c^{2}-1}\right)+\sqrt{c^{2}-1}\left(\left(c-\sqrt{c^{2}-1}\right) z-x\right)}{h(a, b, c)}
\end{array}
$$


Proof. Considering the relation between trace and length of a geodesic, $a, b, c, d$ and $e$ are straightforward. As $s=\frac{1}{2}(\ln (p(a, b, c, d, e, x, z) h(a, b, c))-\ln (h(c, d, e)))$, we have $e^{2 s} \frac{h(c, d, e)}{h(a, b, c)}=p(a, b, c, d, e, x, z)$. We want a point in the Teichmüller space of $\mathrm{X}$ pieces, thus $Q(a, b, c, d, e, x, z)=0$. It remains to prove that there is only one solution to the system of equations, but this is easy, as the set of all solutions (in the $x z$-plane) to the linear equation $p(a, b, c, d, e, x, z)=0$ is an asymptote to the hyperbola defined by $Q(a, b, c, d, e, x, z)=0$ (see the proof of proposition [13).

Remark. We could have explicitly given $x$ and $z$ in terms of Fenchel-Nielsen coordinates, but the terms get very big.

We can now build the generating elements of the modular group in FenchelNielsen coordinates using the fact that $\Psi$ and $\Phi$ are inverse isomorphisms. We proceed as follows:

For a given element $\varphi$ of the modular group in trace coordinates, we construct $\varphi^{\mathrm{F}-\mathrm{N}}:=\Phi \circ \varphi \circ \Psi$ which is an element of the modular group in Fenchel-Nielsen coordinates because $\Psi$ and $\Phi$ are inverse isomorphisms; hence, the following diagram commutes:

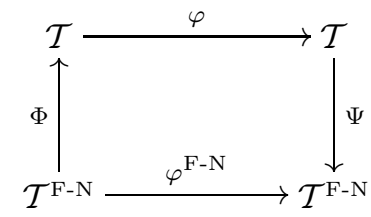

If we apply this procedure to $\varphi_{Y_{a}}, \varphi_{Y_{d}}, \varphi_{i n v}$ and $\varphi_{t u r n}$, we obtain

$$
\begin{aligned}
& \varphi_{Y_{a}}^{\mathrm{F}-\mathrm{N}}:\left(l_{1}, l_{2}, l_{3}, l_{4}, l_{5}, \theta\right) \longmapsto\left(l_{2}, l_{1}, l_{3}, l_{4}, l_{5}, \theta+\pi\right), \\
& \varphi_{Y_{d}}^{\mathrm{F}-\mathrm{N}}:\left(l_{1}, l_{2}, l_{3}, l_{4}, l_{5}, \theta\right) \longmapsto\left(l_{1}, l_{2}, l_{3}, l_{5}, l_{4}, \theta+\pi\right), \\
& \varphi_{i n v}^{\mathrm{F}-\mathrm{N}}:\left(l_{1}, l_{2}, l_{3}, l_{4}, l_{5}, \theta\right) \longmapsto\left(l_{2}, l_{1}, l_{3}, l_{5}, l_{4},-\theta\right) \text {, } \\
& \varphi_{\text {turn }}^{\mathrm{F}-\mathrm{N}}:\left(l_{1}, l_{2}, l_{3}, l_{4}, l_{5}, \theta\right) \longmapsto\left(l_{2}, l_{4}, 2 \operatorname{arccosh}(z), l_{5}, l_{1}, \tau\right) \text {; }
\end{aligned}
$$

where

$$
\tau=2 \pi \frac{\ln \left(p\left(\varphi_{\text {turn }}(a, b, c, d, e, x, z)\right) h(b, d, z)\right)-\ln (h(z, e, a))}{4 \operatorname{arccosh}(z)}
$$

with $(a, b, c, d, e, x, z)=\Phi\left(l_{1}, l_{2}, l_{3}, l_{4}, l_{5}, \theta\right)$.

\section{INTERSECTING GEODESICS}

The fact that the parameterization we chose for the $\mathrm{X}$ piece depends only on lengths of geodesics gives us a powerful tool using simple mathematics to state general results on geodesic lengths on any purely hyperbolic (no elliptic or parabolic elements in its fundamental group) Riemann surface. In this section we give evidence to this by treating the following question: Is there a certain constant independent of the surface such that two geodesics of lengths smaller than it cannot intersect more than once?

Remark. P. Buser treats a similar problem in Bus92 and gives the following result using collars: On a purely hyperbolic Riemann surface, two geodesics shorter than $2 \operatorname{arcsinh}(1)=2 \operatorname{arccosh}(\sqrt{2})$ are pairwise disjoint. 
Lemma 27. Let $(a, b, c, d, e, x, z)$ be an element of the Teichmüller space of Riemann surfaces of signature $(0,4)$. Then $(z-1)(c-1) \geq 4 a_{m}^{2}\left(a_{m}=\min \{a, b, d, e\}\right)$.

Proof. By corollary 12 we know that $Q=0$. The discriminant $\operatorname{disc}(Q, x)$ of $Q$ in respect to $x$ must therefore be positive. But $\operatorname{disc}(Q, x)(z)$ is a quadratic polynomial in $z$ that is negative if evaluated in $1(\operatorname{disc}(Q, x)(1)<0)$. Thus $z$ must be greater than its right root, i.e.

$$
\begin{aligned}
z & \geq \frac{a e+b d+c(a d+b e)+\sqrt{h(a, b, c) h(c, d, e)}}{c^{2}-1} \\
& \geq \frac{2(c+1) a_{m}^{2}+h\left(a_{m}, a_{m}, c\right)}{c^{2}-1}=\frac{4 a_{m}^{2}}{c-1}+1 .
\end{aligned}
$$

But this is equivalent to $(z-1)(c-1) \geq 4 a_{m}^{2}$.

Corollary 28. If, on an $X$ piece, there are two closed geodesics $\gamma$ and $\zeta$ of lengths $l_{1}$ and $l_{2}$ that intersect twice, then $\max \left\{l_{1}, l_{2}\right\}>2 \operatorname{arccosh}(3)$. This bound is sharp.

Remark. In terms of traces, this means that if two elements $\gamma$ and $\zeta$ of a subgroup of $S L(2, \mathbb{R})$ corresponding to an X piece are such that $\max \{\operatorname{tr}(\gamma), \operatorname{tr}(\zeta)\} \leq 3$, then their corresponding geodesics intersect at most once.

Proof. As the smallest self-intersecting geodesics on any Riemann surface is a figure eight geodesic (cf. [Bus92]) and as any figure eight geodesic on a purely hyperbolic Riemann surface is strictly longer than $4 \operatorname{arcsinh}(1)=2 \operatorname{arccosh}(3)$ (cf. [Bus92]), we can suppose that $\gamma$ and $\zeta$ are simple (non-self-intersecting).

As any simple closed geodesic (other than the border geodesics) on an $\mathrm{X}$ piece is dividing, we are in the situation of figure 11

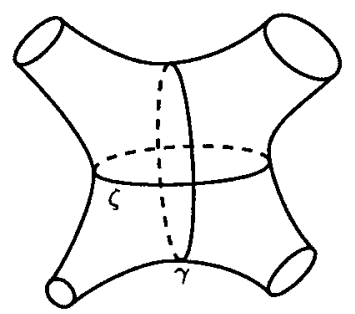

Figure 11. Two intersections on an $\mathrm{X}$ piece

Thus, there is an element $\left(a, b, c=\cosh \left(l_{1} / 2\right), d, e, x, z=\cosh \left(l_{2} / 2\right)\right)$ of Teichmüller space of Riemann surfaces of signature $(0,4)$ corresponding to the $\mathrm{X}$ piece.

Lemma 27implies $(z-1)(c-1)>4$ and thus $\max \{z, c\}>3$ which is equivalent to $\max \left\{l_{1}, l_{2}\right\}>2 \operatorname{arccosh}(3)$.

It is easy to see that for any $\rho>0,\left(1+\frac{\rho}{2}, 1+\frac{\rho}{2}, 3+\rho, 1+\frac{\rho}{2}, 1+\frac{\rho}{2}, 7+4 \rho+\frac{\rho^{2}}{2}, 3+\rho\right)$ is an element of moduli space of surfaces of signature $(0,4)$ that has two geodesics of lengths $2 \operatorname{arccosh}(3+\rho)$ that intersect twice, hence the sharpness of the bound.

Lemma 29. If, on a $Q$ piece (a surface of signature $(1,1)$ ), there are two closed geodesics $\gamma$ and $\zeta$ of lengths $l_{1}$ and $l_{2}$ that intersect twice, then $\max \left\{l_{1}, l_{2}\right\}>$ $2 \operatorname{arccosh}(2)$. This bound is sharp. 
Proof. We will use several results of [BS88], investigating the length spectrum of the one-holed torus. Indeed, in [BS88], the authors establish that the geodesics corresponding to the first three lengths of the spectrum (of simple non-boundary geodesics) intersect only once and are such that their traces $b, m$ and $c$ (i.e. $\cosh ($ lengths $/ 2))$ satisfy $1<b \leq m \leq c \leq b m$ and $\frac{e-1}{2}=c(2 b m-c)-m^{2}-b^{2}$ where $e$ is the trace of the boundary geodesic $\eta$ and $z=2 b m-c$ is the trace of the forth shortest simple non-boundary geodesic.

As $\gamma$ and $\zeta$ intersect twice, the minimum of $\max \{l(\gamma), l(\zeta)\}$ is obtained when they are third and forth shortest and have equal length, i.e. $c=b m=z$, where $c=\operatorname{tr}(\gamma)$ and $z=\operatorname{tr}(\zeta)$. But in that case, $0<\frac{e-1}{2}=b^{2} m^{2}-b^{2}-m^{2} \leq b^{2} m^{2}-2 b m$ and thus $c=z>2$, which is not possible for $c=\cosh \left(l_{1} / 2\right) \leq 2$ and $z=\cosh \left(l_{2} / 2\right) \leq 2$.

It is easy to see that for any $\rho>0,(\sqrt{2+\rho}, \sqrt{2+\rho}, 2+\rho)$ is an element of moduli space of surfaces of signature $(1,1)$ (as established in [BS88]), that has two geodesics of lengths $2 \operatorname{arccosh}(2+\rho)$ that intersect twice, hence the sharpness of the bound.

Lemma 30. Let $\gamma$ and $\zeta$ be two simple closed geodesics on a Riemann surface that intersect more than twice and whose lengths are $l_{1}$ and $l_{2}$. Then there exist two closed geodesics $\gamma^{\prime}$ and $\zeta^{\prime}$ with lengths $l_{1}^{\prime}$ and $l_{2}^{\prime}$ such that $l_{1}^{\prime} \leq l_{1}$ and $l_{2}^{\prime} \leq l_{2}$ and such that either one of $\gamma^{\prime}$ and $\zeta^{\prime}$ is not simple or $\left|\gamma^{\prime} \cap \zeta^{\prime}\right|=2$.

Proof. Put an arbitrary orientation on $\zeta$. Take one intersection point and name it $A$. From $A$ go along $\zeta$ (following the chosen orientation) to the next intersection point, name it $B$, follow $\zeta$ to the next intersection point and name it $C$. Now orient $\gamma$ such that $A B C$ come in that order on it (there might be some intersections between $A$ and $B, B$ and $C, C$ and $A$ ). Considering the collar (stable neighborhood) of $\zeta$, there are now two possible situations:

(1) The $A$ intersection has the same orientation as the $C$ intersection:
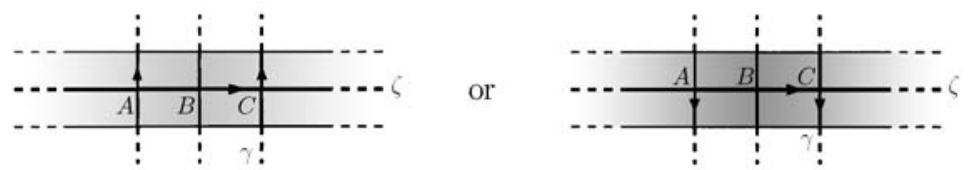

(2) There is no orientation of $\zeta$ and no intersection that we can name $A$ such that we are in the first situation. It is easy to see that in this case there must be an intersection that can be named $A$ and an orientation of $\zeta$ such that the $A$ intersection has the same orientation as the $B$ intersection:

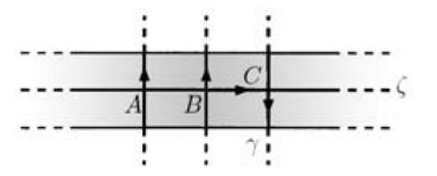

or

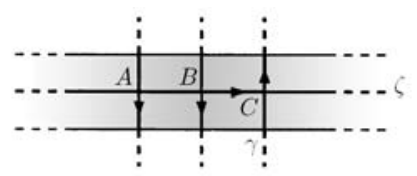

In situation (1), we build the oriented curves $\tilde{\gamma}^{\prime}$ and $\tilde{\zeta}^{\prime}$ as follows:

- If the oriented segment from $C$ to $A$ on $\zeta$ is shorter than the corresponding segment on $\gamma$, we define $\tilde{\zeta}^{\prime}=\zeta$ and $\tilde{\gamma}^{\prime}$ the curve obtained by following $\gamma$ from $A$ to $C$ then $\zeta$ back to $A$. 
- If not, we define $\tilde{\gamma}^{\prime}=\gamma$ and $\tilde{\zeta}^{\prime}$ the curve obtained by following $\zeta$ from $A$ to $C$ then $\gamma$ back to $A$.

In both cases $\tilde{\gamma}^{\prime}$ and $\tilde{\zeta}^{\prime}$ intersect exactly twice and are shorter than $\gamma$ and $\zeta$. Take the geodesics $\gamma^{\prime}$ and $\zeta^{\prime}$ homotopic to $\tilde{\gamma}^{\prime}$ and $\tilde{\zeta}^{\prime}$. They still intersect twice and using the argument that the geodesic is always the shortest curve of its homotopy class (see [FLP79]), we show that $\gamma^{\prime}$ and $\zeta^{\prime}$ are shorter than $\gamma$ and $\zeta$.

In situation (2) we build the oriented curves $\tilde{\gamma}^{\prime}$ and $\tilde{\zeta}^{\prime}$ as follows:

- If the oriented segment from $A$ to $B$ on $\zeta$ is shorter than the corresponding segment on $\gamma$, we define $\tilde{\zeta}^{\prime}=\zeta$ and $\tilde{\gamma}^{\prime}$ the curve obtained by following $\gamma$ from $B$ to $A$ then $\zeta$ back to $B$.

- If not, we define $\tilde{\gamma}^{\prime}=\gamma$ and $\tilde{\zeta}^{\prime}$ the curve obtained by following $\zeta$ from $B$ to $A$ then $\gamma$ back to $B$.

In both cases $\tilde{\gamma}^{\prime}$ and $\tilde{\zeta}^{\prime}$ and thus the homotopic geodesics $\gamma^{\prime}$ and $\zeta^{\prime}$ are shorter than $\gamma$ and $\zeta$. If we are in the second case, and if there is an intersection between $A$ and $B$ on $\gamma$, then $\zeta^{\prime}$ is not simple. In every other case, we have diminished the intersection number by exactly one.

Iterating this procedure, we get two geodesics that are shorter and either intersect one another twice or have self-intersections.

Proposition 31. If, on a purely hyperbolic Riemann surface, there are two closed geodesics $\gamma$ and $\zeta$ of lengths $l_{1}$ and $l_{2}$ both smaller than (or equal to) $2 \operatorname{arccosh}(2)$, then they intersect at most once. This bound is sharp.

Remark. In terms of traces, this means that if two elements $\gamma$ and $\zeta$ of a subgroup of $S L(2, \mathbb{R})$ corresponding to a Riemann surface are such that $\max \{\operatorname{tr}(\gamma), \operatorname{tr}(\zeta)\} \leq 2$, then their corresponding geodesics intersect at most once.

Proof. For the first part of the proposition, we suppose that $\gamma$ and $\zeta$ intersect more than once and show that this implies that $\max \left\{l_{1}, l_{2}\right\}>2 \operatorname{arccosh}(2)$. The $\operatorname{second}$ part is proven by the sharpness of the bound for Q pieces (lemma 29).

As in the proof of corollary 28, we can suppose that $\gamma$ and $\zeta$ are simple (otherwise they are longer than $4 \operatorname{arcsinh}(1)=2 \operatorname{arccosh}(3)$, cf. [Bus92]).

Suppose now that $\gamma$ and $\zeta$ intersect exactly twice, call their intersections $A$ and $B$, and give them an arbitrary orientation. Considering the collar of $\zeta$, there are two possible situations (up to orientation):

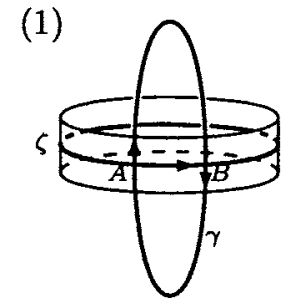

(2)

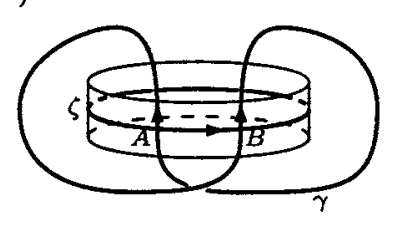

(1) In the first situation, we build the oriented curves $\tilde{\alpha}, \tilde{\beta}, \tilde{\delta}$ and $\tilde{\varepsilon}$ :

- For $\tilde{\alpha}$, we follow $\gamma^{-1}$ from $A$ to $B$, then back to $A$ on $\zeta$.

- For $\tilde{\beta}$, we follow $\zeta^{-1}$ from $A$ to $B$, then back to $A$ on $\gamma^{-1}$.

- For $\tilde{\delta}$, we follow $\gamma$ from $A$ to $B$, then back to $A$ on $\zeta^{-1}$.

- For $\tilde{\delta}$, we follow $\zeta$ from $A$ to $B$, then back to $A$ on $\gamma$. 
None of these curves are null-homotopic because otherwise $\gamma$ and $\zeta$ would be homotopic to two other geodesics intersecting at most once, but in each homotopy class there is only one geodesic (cf. [FLP79]). The geodesics $\alpha, \beta, \delta$ and $\varepsilon$ homotopic to $\tilde{\alpha}, \tilde{\beta}, \tilde{\delta}$ and $\tilde{\varepsilon}$ may be the same but otherwise do not intersect because we can build the homotopic curves $\bar{\alpha}, \bar{\beta}, \bar{\delta}$ and $\bar{\varepsilon}$ that do not intersect:

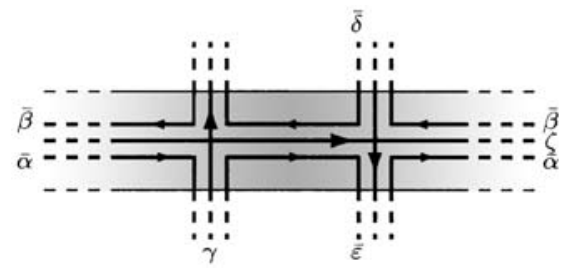

We can now cut the surface along the geodesics $\alpha, \beta, \delta$ and $\varepsilon$ and get a surface that is a Nielsen kernel of an X piece containing $\gamma$ and $\zeta$. By corollary 28 we know that $\max \left\{l_{1}, l_{2}\right\}>2 \operatorname{arccosh}(3)$.

(2) In the second situation, we build the oriented curves $\tilde{\alpha}$ and $\tilde{\beta}$ :

- For $\tilde{\alpha}$, we follow $\gamma^{-1}$ from $A$ to $B$, then $\zeta^{-1}$ back to $A$, then $\gamma$ to $B$ and finish on $\zeta$ back to $A$.

- For $\tilde{\beta}$, we follow $\zeta^{-1}$ from $A$ to $B$, then $\gamma^{-1}$ back to $A$, then $\zeta$ to $B$ and finish on $\gamma$ back to $A$.

One of these curves may be null-homotopic, but not both because otherwise the surfaces would be a torus as can be seen cutting the surface along $\zeta$ and from $A$ to $B$ along $\gamma$ and tracing the curves $\bar{\alpha}$ and $\bar{\beta}$ homotopic to $\tilde{\alpha}$ and $\tilde{\beta}$ and (if they are not null-homotopic) to the geodesics $\alpha$ and $\beta$ as follows:

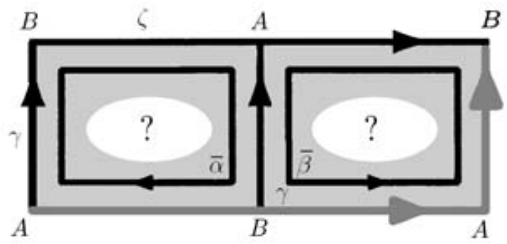

If one of $\bar{\alpha}$ and $\bar{\beta}$ is null-homotopic (say $\bar{\beta} \sim 0$ ), we cut along the geodesic other geodesic (in our case $\alpha$ ) and get a surface that is the Nielsen kernel of a $\mathrm{Q}$ piece and contains both $\gamma$ and $\zeta$ (see figure 12). We conclude by lemma 29] knowing that $\max \left\{l_{1}, l_{2}\right\}>2 \operatorname{arccosh}(2)$ in this case.
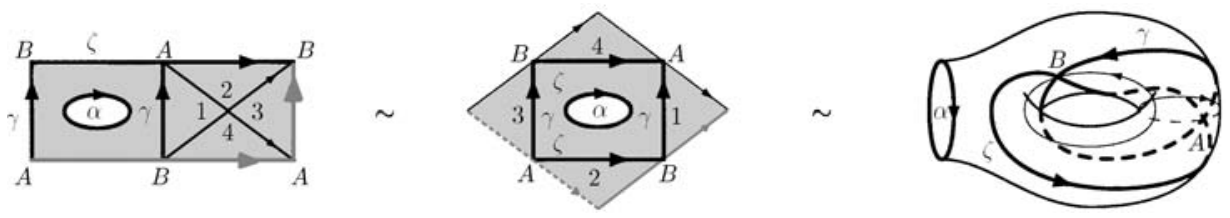

Figure 12. Situation of a $\mathrm{Q}$ piece

If none of $\bar{\alpha}$ and $\bar{\beta}$ is null-homotopic, we cut the original surface along the geodesics $\alpha$ and $\beta$ and get a surface that is the Nielsen kernel of a Fish piece (a surface of signature $(1,2))$ and contains both $\gamma$ and $\zeta$ (see figure 13). 


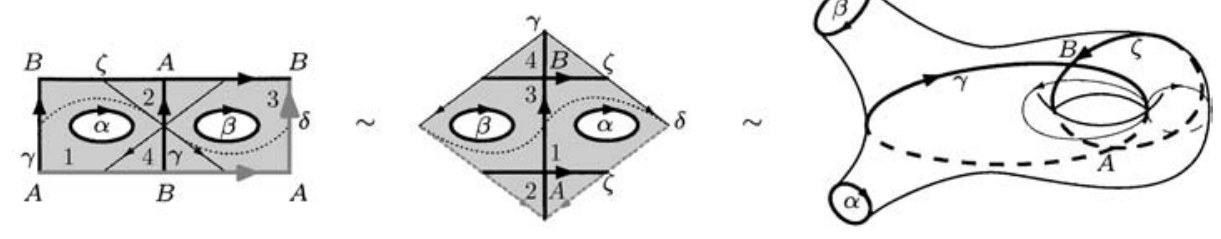

Figure 13. Situation of a fish piece

Let $\delta$ be a geodesic intersecting $\gamma$ twice, such that $\alpha, \delta$ and $\zeta$ are the border geodesics of a $\mathrm{Y}$ piece (dotted in figure 13). If we cut along $\delta$, we get an $\mathrm{X}$ piece with boundary geodesics $\alpha, \beta, \delta$ and a copy of $\delta$ that has $\zeta$ as a dividing geodesic. Let $\eta$ now be another dividing geodesic that does not intersect the geodesic arcs constituting $\gamma$. Let $z=\cosh (l(\zeta) / 2), a=\cosh (l(\alpha) / 2), b=\cosh (l(\beta) / 2), d=$ $\cosh (l(\delta) / 2)$ and $e=\cosh (l(\eta) / 2)$. By the proof of lemma [27] we know that

$$
\begin{aligned}
z & \geq \frac{d(a+b)(e+1)+\sqrt{h(a, b, e)(e+1)\left(e-1+2 d^{2}\right)}}{e^{2}-1} \\
& >\frac{2 d+\sqrt{(e+1)\left(e-1+2 d^{2}\right)}}{e^{2}-1} .
\end{aligned}
$$

This quantity is minimal for $\delta$, the forth shortest geodesic, and $\gamma$, the third shortest geodesic on the Nielsen kernel of a $\mathrm{Q}$ piece (obtained by cutting apart the original surface along $\eta$ ) because $\gamma$ and $\delta$ intersect each other twice and we want $\gamma$ to be shorter than $\delta$ (otherwise $c=\cosh (l(\gamma) / 2)>2$ by lemma 27). But on this Q piece we have $\frac{e-1}{2}=c d-m^{2}-n^{2}<d(c-1)$ because $c=2 m n-d$ and thus $(m-n)^{2}<m^{2}+n^{2}-d$ (cf. proof of lemma 29] and [BS88]). This implies

$$
\begin{aligned}
z>\frac{2 d+\sqrt{(e+1)\left(e-1+2 d^{2}\right)}}{e^{2}-1} & =\frac{d}{\frac{e-1}{2}}+\sqrt{\frac{e+1}{e-1}\left(1+\frac{d^{2}}{\frac{e-1}{2}}\right)} \\
& >\frac{1}{c-1}+\sqrt{1+\frac{d}{c-1}}>2 \text { if } c<2,
\end{aligned}
$$

which proves the proposition.

\section{REFERENCES}

[Art47] E. Artin, Braids and permutations, Ann. of Math. (2) 48 (1947), 643-649. MR0020989 $(9: 6 \mathrm{c})$

[Bea83] A. F. Beardon, The geometry of discrete groups, Graduate Texts in Mathematics, vol. 91, Springer-Verlag, New York, 1983. MR 0698777 (85d:22026)

[BG99] R. L. Benedetto and W. M. Goldman, The topology of the relative character varieties of a quadruply-punctured sphere, Experiment. Math. 8 (1999), no. 1, 85-103. MR1685040 (2000c:57028)

[Bin00] A. Binotto, Surfaces de riemann de type (0,3) et (1,1): polynômes au service de l'étude spectrale, Ph.D. thesis, École Polytechnique Fédérale de Lausanne, 2000.

[BS88] P. Buser and K.-D. Semmler, The geometry and spectrum of the one-holed torus, Comment. Math. Helv. 63 (1988), no. 2, 259-274. Mr0948781 (89k:58286)

[Bus92] P. Buser, Geometry and spectra of compact Riemann surfaces, Progress in Mathematics, vol. 106, Birkhäuser Boston Inc., Boston, MA, 1992. MF 1183224 (93g:58149)

[FLP79] A. Fathi, F. Laudenbach, and V. Poénaru, Travaux de Thurston sur les surfaces, Astérisque, vol. 66, Société Mathématique de France, Paris, 1979, Séminaire Orsay. 
[FR95] B. Fine and G. Rosenberger, Classification of all generating pairs of two generator Fuchsian groups, Groups '93 Galway/St. Andrews, Vol. 1 (Galway, 1993), London Math. Soc. Lecture Note Ser., vol. 211, Cambridge Univ. Press, Cambridge, 1995, pp. 205-232. MR1342792 (96i:20065)

[Hel74] H. Helling, Uber den Raum der kompakten Riemannschen Flächen vom Geschlecht 2, J. Reine Angew. Math. 268/269 (1974), 286-293, Collection of articles dedicated to Helmut Hasse on his seventy-fifth birthday, II. MR0361167 (50:13613)

[Kee65] Linda Keen, Canonical polygons for finitely generated Fuchsian groups, Acta Math. 115 (1965), 1-16. MR0183873 (32:1349)

[Kee66] Intrinsic moduli on Riemann surfaces, Ann. of Math. (2) 84 (1966), 404-420. MR0203000 (34:2859)

[Kee71] , On Fricke moduli, Advances in the Theory of Riemann Surfaces (Proc. Conf., Stony Brook, N.Y., 1969), Ann. of Math. Studies, No. 66. Princeton Univ. Press, Princeton, N.J., 1971, pp. 205-224. MR0288252(44:5450)

[Kee73] - A correction to: "On Fricke moduli", Proc. Amer. Math. Soc. 40 (1973), 60-62. $\operatorname{MR} 0320306(47: 8845)$

[Kee77] _ A rough fundamental domain for Teichmüller spaces, Bull. Amer. Math. Soc. 83 (1977), no. 6, 1199-1226. MR0454075 (56:12326)

[KR78] R. N. Kalia and G. Rosenberger, Automorphisms of the Fuchsian groups of type (0; 2, 2, 2, q; 0), Comm. Algebra 6 (1978), no. 11, 1115-1129. MR0498884 (80a:10039)

[Luo98] Feng Luo, Geodesic length functions and Teichmüller spaces, J. Differential Geom. 48 (1998), no. 2, 275-317. MR1630186 (99e:32031)

[Mag80] W. Magnus, Rings of Fricke characters and automorphism groups of free groups, Math. Z. 170 (1980), no. 1, 91-103. MR.0558891 (81a:20043)

[Mas65] B. Maskit, On Klein's combination theorem, Trans. Amer. Math. Soc. 120 (1965), 499509. MR0192047 (33:274)

[Mas88] _ Kleinian groups, Grundlehren der Mathematischen Wissenschaften [Fundamental Principles of Mathematical Sciences], vol. 287, Springer-Verlag, Berlin, 1988. MR0959135 (90a:30132)

[Sem88] K.-D. Semmler, A fundamental domain for the Teichmüller space of compact Riemann surfaces of genus 2, Ph.D. thesis, École Polytechnique Fédérale de Lausanne, 1988.

[SS86] M. Seppälä and T. Sorvali, Parametrization of Möbius groups acting in a disk, Comment. Math. Helv. 61 (1986), no. 1, 149-160. MR0847525 (87i:20089)

[SS88] _ Parametrization of Teichmüller spaces by geodesic length functions, Holomorphic functions and moduli, Vol. II (Berkeley, CA, 1986), Math. Sci. Res. Inst. Publ., vol. 11, Springer, New York, 1988, pp. 267-284. MR0955845 (89m:32040)

[SS89] _ Affine coordinates for Teichmüller spaces, Math. Ann. 284 (1989), no. 1, 165176. MR0995388 (90e:32024)

[SS92] _ Geometry of Riemann surfaces and Teichmüller spaces, North-Holland Mathematics Studies, vol. 169, North-Holland Publishing Co., Amsterdam, 1992. MR1202043 (94b:32034)

EPFl SB IGAT GEOM, BÂtiment MA, Station 8, CH-1015 Lausanne (Switzerland)

E-mail address: thomas.gauglhofer@epfl.ch

EPFL SB IGAT GEOM, BÂtiment MA, Station 8, CH-1015 Lausanne (Switzerland)

E-mail address: klaus-dieter.semmler@epfl.ch 